\title{
a2-Macroglobulin-Rich Serum As a Master Inhibitor of Inflammatory Factors Attenuates Cartilage Degeneration in a Mini Pig Model of Osteoarthritis Induced By "Idealized" Anterior Cruciate Ligament Reconstruction
}

\section{Ruipeng Zhao}

The Second Hospital of Shanxi Medical University

\section{Xiaochun Wei}

the second hospital of shanxi medical unversity

\section{Chengming Zhang}

The Second Hospital of Shanxi Medical University

\section{Hongru Wu}

Shanxi Institute of sports science

\section{Chuan Xiang}

the second hospital of shanxi medical unversity

Haoqian Li

Second Hospital of Shanxi Medical University

\section{Wangping Duan}

Second Hospital of Shanxi Medical University

\section{Zhiqing Duan}

Department of Biochemistry and Molecular Biology ,Shanxi medical vnversity

Chuanjiang Li

Second Hospital of Shanxi Medical University

\section{Yu Zhao}

The Second Hospital of Shnxi Medical University

\section{Lingan Huang ( $\nabla$ huang_3469@163.com )}

The Second Hospital of Shanxi Medical University

\section{Research article}

Keywords: Osteoarthritis, a2M-rich serum, anterior cruciate ligament, articular cartilage, inflammation, mini pig 
Posted Date: November 10th, 2021

DOI: https://doi.org/10.21203/rs.3.rs-1043859/v1

License: (c) (i) This work is licensed under a Creative Commons Attribution 4.0 International License. Read Full License 


\section{Abstract}

Background: a2-Macroglobulin ( $\mathrm{a} 2 \mathrm{M}$ ) is important for chondral protection in post-traumatic osteoarthritis (PTOA). However, its injection into xenogeneic joint cavities has safety hazards, limiting clinical applications. Exploring serum a2M-enriching strategies and the therapeutic effect and mechanism of a2M-rich serum (a2MRS) autologous joint injection to treat PTOA has significant value.

Methods: A unique filtration process was used to concentrate a2M from serum. Human osteoarthritic chondrocytes induced with interleukin (IL)-1 $\beta$ were used to evaluate catabolic enzymes, cell proliferation, apoptosis, and gene expression $24 \mathrm{~h}$ after a2MRS treatment. Eighteen mature female mini pigs were randomized to three groups, sham $(n=6)$, "idealized" anterior cruciate ligament autograft reconstruction $(I A C L-R)(n=6)$, and IACL-R+a2MRS $(n=6)$. Expression of inflammatory factors in the synovial fluid (SF) was measured using Luminex assays. Gait features were recorded using the Tekscan Walkway system. The extent of PTOA progression was evaluated using imaging, real-time PCR, and histology 3 months post-surgery.

Results: The a2M concentration in a2MRS was higher than that in human and mini pig serum, respectively. In vitro, a2MRS significantly promoted human chondrocyte proliferation $(p<0.001)$ and reduced apoptosis $(p<0.001)$ and chondrocyte catabolic cytokine gene transcription $(p<0.001)$ and secretion $(p<0.001)$. In vivo, SF concentrations of all tested inflammatory factors were significantly lower in the IACL-R+a2MRS group than in the IACL-R group $(p<0.001)$. All gait parameters in the IACL$R+a 2 M R S$ group returned to normal significantly early compared to those in the IACL-R group $(p<0.05)$. Imaging , histology, and biochemistry data showed that cartilage degeneration in the IACL-R+a2MRS group was significantly diminished relative to that in the IACL-R group $(p<0.001)$.

Conclusion: Injecting a2MRS into the joint cavity after IACL-R can significantly delay articular cartilage degeneration.

\section{Background}

Anterior cruciate ligament $(\mathrm{ACL})$ rupture, one of the most common joint injuries in young people, is conventionally treated using surgical ACL reconstruction (ACL-R). However, even with the best surgical techniques available, these patients remain at a high risk for post-traumatic osteoarthritis (PTOA)[1-3]. Recently, researchers developed an "idealized" ACL autograft reconstruction (IACL-R) model[4, 5]. Notably, the authors found that cartilage degeneration still occurred despite this reconstruction and concluded that there was a significant correlation between the expression of inflammatory factors and cartilage injury. Moreover, other studies have indicated that catabolic proteases and cytokines reach their peak levels within $48 \mathrm{~h}$ after joint injury, initiating cell death and cartilage matrix degeneration[6-8]. Thus, early intervention to reduce the expression of these catabolic proteases and cytokines is critical to prevent or delay cartilage degeneration. 
a2-macroglobulin (a2M), a tetrameric macromolecular glycoprotein, is mainly synthesized and secreted into the body fluids by liver epithelial parenchymal cells[9]. To date, a2M has been shown to play an important role in the diagnosis of diseases, prediction of liver fibrosis staging[10], non-invasive diagnosis of type II diabetes[11], and the treatment of various diseases including alleviating pain in subacromial bursitis, lateral epicondylitis, Achilles tendonitis, spinal intervertebral discogenic[12, 13] and jaw osteoradionecrosis[14]. Moreover, some studies have demonstrated that supplemental intra-articular a2M provides chondral protection in PTOA[15-17]. However, a2M is expensive. More importantly, the longterm injection of a2M, a blood protein component, into the xenogeneic joint cavity has safety hazards, such as immune rejection, which limits its clinical application. In the current study, we aimed to develop and use a2M-rich serum (a2MRS) to treat cartilage degeneration following IACL-R. We hypothesized that a2MRS could significantly reduce the expression of inflammatory factors in synovial fluid (SF), promote early recovery of the gait, and effectively attenuate cartilage degeneration.

\section{Materials And Methods}

\section{Study Design}

All procedures in this study were approved by the Ethics Committee of the Second Hospital of Shanxi Medical University (NO. SYDL2019001) and conformed to the ARRIVE guidelines. The mini pigs were purchased from the Beijing Shichuang Century Mini pig Breeding Base (Certificate number:

SCXX(jing)2013-0008). Eighteen mature female mini pigs (age, $18 \pm 1.55$ months; weight, $43.3 \pm 3.67 \mathrm{~kg}$ ) were randomized into three groups based on animal ear numbers: sham $(n=6)$, IACL-R $(n=6)$, and IACL$R+a 2 M R S(n=6)$. Unilateral surgery was performed on the right hind limbs of all mini pigs. All animals were housed at the China Institute for Radiation Protection (Certificate number: SYXK(Jin)2016-0002). All animals were euthanized with a pentobarbital overdose 3 months after surgery. Specific information on animal care can be found in the supplementary text (Text 1). All a2MRS used in in vitro experiments were derived from human serum and in vivo from autologous serum of mini pigs. All subjective scores were independently evaluated by two experienced examiners, who were blinded to the animal number and experimental condition.

\section{a2M Concentrate from Human Serum}

Whole blood $(13 \mathrm{ml})$ was collected in a coagulation tube and centrifuged at 2,000 ' $g$ for 20 min to obtain $6 \mathrm{ml}$ of serum, which was then added to the upper filter of the ultrafiltration tube (Cytonics Corporation, West Palm Beach, Florida, USA). The upper concentrate was obtained under different conditions of centrifugal force $\left(3000,4000\right.$, and $5000^{\prime} \mathrm{g}$ ) and time (20,30, and $\left.40 \mathrm{~min}\right)$. Finally, the best concentration conditions were determined based on the concentration of a2M in the upper concentrate.

\section{Human Chondrocyte Isolation and Primary Culture}

Human chondrocytes were isolated as previously described[18] and plated in 6-well culture plates at a density of $1 \times 10^{6}$ cells/plate. At $90 \%$ confluence, the cells were cultured overnight under serum-free 
conditions and then treated with $10 \mathrm{ng} / \mathrm{ml}$ recombinant human interleukin (IL)- $1 \beta$ for $2 \mathrm{~h}$ before treatment with a2MRS. It was ensured that the concentration of a2M was $0.25 \mathrm{mg} / \mathrm{ml}$ in the culture medium. The culture medium and chondrocytes were collected and analyzed.

\section{Elisa Assays}

a2M concentrations in the upper concentrate under different centrifugal conditions were determined using ELISA (EK1118, Boster Bio, China). The human chondrocyte culture medium was collected 24h after a2MRS treatment and analyzed for the presence of matrix metalloproteinase 13 (MMP-13), tumor necrosis factor-a (TNF-a), and IL-6 using ELISA.

\section{Human Chondrocyte Proliferation and Apoptosis Assays}

Human chondrocyte proliferation was detected at $0,24,36$, and $48 \mathrm{~h}$ using the Cell Counting Kit-8 (CCK-8) cell viability assay kit ( Boster Biological Technology, China). Human chondrocytes were collected $24 \mathrm{~h}$ after a2MRS treatment and apoptosis was detected using a Terminal transferase dUTP end Labeling (TUNEL) assay kit (Key GEN Bio TECH, China). The percentage of positive cells was determined. The detailed procedure was in accordance with the manufacturer's protocol.

\section{RNA Isolation and Real-time PCR Assays}

mRNA levels of col-2, aggrecan, MMP-3, and MMP-13 in human chondrocyte samples and those plus col$10 a 1$ and Runx-2 in minipig cartilage weight-bearing sites of the medial tibial plateau (MTP) were measured by real-time PCR. Primer pairs are listed in Supplementary Table 1. Levels of gene expression were normalized to $18 \mathrm{~S}$ rRNA expression. The data were analyzed using the comparison $\mathrm{Ct}\left(2^{-\Delta \Delta C t}\right)$ method and expressed as the fold-change relative to the respective control. The detailed PCR procedure previously described.[19]

\section{Mini Pig a2MRS Reserve}

With the help of a veterinarian, $120 \mathrm{ml}$ of whole blood was collected from the anterior vena cava of each mini pig into coagulation tubes. According to the best concentration conditions (centrifugal force: 5000 ' $g$, time: $30 \mathrm{~min}$ ), $12-15 \mathrm{ml}$ of a2MRS was obtained per mini pig (marked according to the mini pig ear number), and these samples were frozen at $-80^{\circ} \mathrm{C}$.

\section{Idealized ACL Autograft Reconstruction Surgery}

All surgeries were performed under anesthesia via an intramuscular injection of $25 \mathrm{mg} / \mathrm{ml}$ tiletamine and $25 \mathrm{mg} / \mathrm{ml}$ zolazepam (Zoletil 50, $1 \mathrm{ml} / 15 \mathrm{~kg}$; Virbac Group, Carros, France). Mini pigs in the IACL-R and IACL-R+a2MRS groups were subjected to surgery based on the methods described by researchers $[4,5]$ (Fig 1). The minipigs in the sham group underwent arthrotomy, temporary patellar dislocation, and coring of one-third of the length of the lateral femoral condyle. 
IACL-R and IACL-R+a2MRS groups. A: The stifle joint was open and the patella was dislocated to expose the ACL (arrow). B: The ACL reconstruction guide was positioned at a $45^{\circ}$ angle (arrow) to the longitudinal axis of the femur. C: Before the hollow drill was about to penetrate the femoral tunnel, a Kirschner wire (diameter $1 \mathrm{~mm}$ ) was drilled along outer edge of the tunnel to prevent the cartilage from splitting. D: The tunnel was gently penetrated by the same diameter thin-walled ring osteotomy to completely severv tendon-bone segment $\mathrm{E}$ : The tendon-bone segment was completely freed (arrow). $\mathrm{F}$ : The tendon-bone segment was fixed in situ with two crossed Kirschner wires.

\section{SF Collection}

SF from the right hind limbs of all animals was collected preoperatively (day 0 ) and postoperatively on days $3,6,14,29$, and 90 . The detailed procedure previously described.[20]

\section{Intra-articular Injections}

Under general anesthesia, intra-articular injections were administered 2, 6, 14, and 29 days post-surgery using a sterile syringe. Under aseptic conditions, $2.5 \mathrm{ml}$ autologous a2MRS was injected into the right hind limbs of mini pigs in the IACL-R+a2MRS group on the indicated days. Animals in the sham and IACL$\mathrm{R}$ groups were administered an equivalent volume of saline.

\section{Luminex Assay}

The Millipore Porcine Cytokine Magnetic Bead Panel (EMD Millipore, No. PCYTMAG-23K) was used to measure the levels of IL-1a, IL-1 3 , IL-2, IL-6, IL-8, IL-18, TNF- $a$, and granulocyte-macrophage colonystimulating factor( GM-CSF). Luminex assays were performed as previously described[5].

\section{Gait Assessment}

Six gait indicators related to biomechanics-maximum force, contact area, peak force, impulse, stance time, and swing time-were determined using the Tekscan Walkway system[21, 22] (Tekscan Inc., USA). To rule out individual differences in learning skills and walking states, each animal was subjected to over 10 successful training sessions per day for 10 consecutive days before the surgery, and all indicators were expressed as a ratio of the average values for the left hind limb divided by the average values for the right hind limb [23]. Gait data were collected preoperatively (day 0 ) and postoperatively on days 7, 15, 30, $45,60,75$, and 90 . All the results obtained are the average of five successful repeated walkway trials performed at each time point for each animal.

\section{Imaging Assessment}

Three months after the surgery, the mini pigs were euthanized with a pentobarbital overdose, and their right hind limbs were severed from the hip joint. Each right hind limb semi flexed was immediately subjected to X-ray examination, computed tomography (CT), three-dimensional CT reconstruction (3D 
$\mathrm{CT}$ ), and magnetic resonance imaging (MRI). The specific imaging parameters are listed in the supplementary text (Text 2).

We determined the Kellgren-Lawrence grade of the right hind limb of each animal by examining the X-ray image[24, 25]. The CT values and thickness of the subchondral bone plate were determined[26]. To avoid interference by metal artifacts, we obtained CT scans of only the middle sagittal plane from the medial femoral condyle (MFC) and MTP of the right hind limb. We also determined the whole-organ MRI score (WORMS) of the MFC and MTP of the right hind limb[27, 28].

\section{Macroscopic Cartilage and Osteophyte Assessment}

Macroscopic damage to the articular cartilage surfaces and osteophyte formation on the MFC, MTP, lateral femoral condyle, lateral tibial plateau, and trochlea were scored according to the Osteoarthritis Research Society International (OARSI) recommendations for sheep and goats[29]

\section{Histological Assessment}

Cartilage samples were obtained by drilling ( $\varphi 8 \mathrm{~mm}$; MOC Medizinische Gerate Gmbh, Fedderingen, Germany) the weight bearing site of the MFC. The cartilage tissue sections $(6 \mu \mathrm{m})$ were stained with safranin $\mathrm{O}$ and fast green as previously described and scored according to the recommendations of OARS[29]. Furthermore, we collected synovium samples from inside the joint capsule. Synovium sections $(4 \mu \mathrm{m})$ were stained with hematoxylin and eosin (H\&E) as previously described and scored according to the OARSI recommendations[29]. Vertical meniscus slices from the middle region of the medial meniscus were processed and stained using H\&E and scored according to the protocol detailed by Pauli et al[30].

\section{Immunohistochemical Assessment}

Cartilage tissue sections from the MFC were used to detect the distribution of collagen-2 (Col-2】ab34712, Abcam), MMP-3 (bs-0413R, Bioss), MMP-9 (bs-4593R, Bioss), MMP-13 (K009743P, Solarbio), Col-10 (bs0554R, Bioss), and Runx-2 (ab76956, Abcam). Quantitative immunohistochemical analysis was performed using an imaging analyzer. The detailed immunohistochemical procedure previously described[31]

\section{Statistical Analysis}

SPSS statistical software (version 13.0) was used to analyze the collected data. Differences in gaits and inflammatory factor levels between the preoperative (day 0 ) and postoperative time points in the same group were analyzed using multiple comparisons of repeated measurement data. Differences in human chondrocytes, minipig gait, inflammatory factor levels, CT values, thicknesses of the subchondral bone plate T2 values, and quantitative immunohistochemical analysis at the same time point among the different groups were estimated using one-way analysis of variance. Differences in macroscopic cartilage and osteophyte scores; microscopic cartilage, synovium, and meniscus scores; and WORMS were 
estimated using nonparametric tests (Wilcoxon rank-sum test). Differences in Kellgren-Lawrence grades were estimated using Fisher probabilities. Statistical significance was determined at $P<0.05$.

\section{Results}

\section{Concentration Analysis of a2M in Different Concentrates From Human and Mini pig}

As the centrifugal force increased and centrifugation time was prolonged, Human a2M concentrations in the upper concentrate correspondingly increased (Table 1). Considering different factors, such as protein biological activity and concentrate volume, the concentration effect of a2M under the conditions of centrifugation at $5000 \times g$ for 30 min was ideal. For human a2MRS, the concentration of a2M was 11.13 $\mathrm{mg} / \mathrm{ml}$, which was 4.88 -fold higher than that in normal human serum. In mini pigs, the concentration of a2M was $12.32 \mathrm{mg} / \mathrm{ml}$, which was 6.48-fold higher than that in normal pig serum (Fig. 2A).

Table 1 Human a2M concentrations in the upper concentrate under different centrifugal conditions $\square \mathrm{mg} / \mathrm{mlauMean} \pm \mathrm{SD} \square \mathrm{n}=6 \square$

\begin{tabular}{|lclc|}
\hline & $3000 \mathrm{~g}$ & $4000 \mathrm{~g}$ & $5000 \mathrm{~g}$ \\
\hline $20 \mathrm{~min}$ & $4.82 \pm 0.75$ & $6.98 \pm 1.15$ & $8.23 \pm 1.12$ \\
$30 \mathrm{~min}$ & $6.46 \pm 0.89$ & $9.22 \pm 1.08$ & $11.13 \pm 0.90$ \\
$40 \mathrm{~min}$ & $7.39 \pm 0.98$ & $9.92 \pm 1.17$ & $11.93 \pm 1.53$ \\
\hline
\end{tabular}

\section{Human Chondrocyte Culture Medium Analysis}

ELISA results demonstrated that exogenous a2MRS significantly inhibited the induction of MMP-13 (P = $0.001)$, TNF- $\alpha(P=0.005)$, and IL-6 $(P<0.001)$ activity in IL-1 $\beta$-induced human primary osteoarthritis chondrocytes (Fig. 2B-D).

\section{Human Chondrocyte Proliferation and Apoptosis Analysis}

a2MRS promoted the proliferation and reduced the apoptosis of human chondrocytes in vitro. The results of the CCK-8 assay showed that the viability of chondrocytes was higher in the IL-1 $\beta+$ a2MRS group than in the IL-1 $\beta$ group $(P<0.001)$, and the viability gradually increased with treatment time (Fig. $2 E)$. The results of the TUNEL assay showed that apoptosis was significantly reduced in the IL-1 $\beta+$ a2MRS group $(18.33 \% \pm 5.71 \%)$ relative to that in the IL-1 $\beta$ group $(32.33 \% \pm 7.23 \%)(P<0.001 ; F i g .2 F, G)$.

\section{Real-time PCR Analysis}


Real-time PCR data indicated that supplementation with a2MRS reduced cartilage matrix catabolism and enhanced anabolic metabolism in vitro (Fig.3A-D) and in vivo (Fig.3E-J). mRNA levels of MMP-3 $(\mathrm{P}<$ 0.001), MMP-13 ( $P<0.001)$, Col-10a1 ( $P=0.001)$, and Runx-2 $(P<0.001)$ were lower in IL-1 $\beta+a 2 M R S$ and IACL-R+a2MRS groups than in IL-1 $\beta$ and IACL-R groups. In contrast, mRNA levels of $C o l-2(P<0.001)$ and aggrecan $(P<0.001)$ showed the opposite pattern. Both were increased in IL-1 $\beta+a 2 M R S$ and IACL$R+a 2 M R S$ groups as compared to levels inIL-1 $\beta$ and IACL-R groupsrespectively.

\section{Inflammatory Factor Analysis}

Postoperatively, changes in the levels of inflammatory factors in different groups, except IL-18 in the IACL-R group, showed similar trends; the levels markedly increased in the early stage and then decreased significantly. IL-2 concentration in the IACL-R group subsequently showed an increasing trend from 30 to 90 day. The concentrations of inflammatory factors, including IL-1 $1 \beta, \mathrm{IL}-6, \mathrm{IL}-18, \mathrm{TNF}-\mathrm{a}$, and GM-CSF, in the IACL-R and IACL-R + a2MRS groups after surgery wer significantly higher than those before surgery $(P<$ 0.001). The concentration of all tested inflammatory factors other than IL-1 $\mathrm{a}$ after surgery was significantly lower in the IACL-R + a2MRS group than in the IACL-R group, and significant difference in peak concentration was observed in all factors $(P<0.001)$. Moreover, the peak concentrations of all detected inflammatory factors, other than IL-18 in the IACL-R group, appeared within 3-14 days after surgery (Fig. 4).

\section{Gait Assessment}

Across all groups, the ratios of all gait parameters of the left hind limb to those of the right hind limb initially showed an increasing trend, followed by a decreasing trend, except in the IACL-R group, which showed another increasing trend toward the end. In the IACL-R group, no gait parameters, from day 45 until day 75 , differed significantly from their values on day $0(P>0.05)$. In the IACL-R + a2MRS group, no gait parameters, from day 30 until euthanasia, differed significantly from their values on day $0(P>0.05)$ (Fig. 5).

The ratios of left hind limb to right hind limb of all gait parameters were similar and close to 1 for symmetry and did not differ significantly in the all groups before surgery $(P>0.05)$. Meanwhile, the postoperative ratios of left hind limb to right hind limb of the gait parameters were significantly greater than 1 in all groups, and the values in the IACL-R group were significantly greater than those in the other two groups other than swing time, especially on days 7 and $15(P<0.001)$. The ratios of left hind limb to right hind limb of the gait parameters was close to 1 on days 45 and 60 , indicating that there were no significant differences among the groups $(P>0.05)$. On day 75 , this ratio increased only in the IACL-R group whereas it remained constant in the sham and IACL-R + a2MRS groups. On day 90 , all gait parameters other than swing time $(P=0.345)$ significantly differed between the IACL-R group and the other two groups $(P<0.001$; Fig. 5$)$. 


\section{Imaging Assessment}

The X-ray examinations showed that joint degeneration in the IACL-R group was relatively noticeable. The joints had a blurred border and mild osteophyte formation compared with those in the IACL-R + a2MRS group (Fig. 6A). The Kellgren-Lawrence grades did not significantly differ among the three groups $(P>$ 0.05; Table 2). Three-dimensional CT reconstruction showed that all joint surfaces were relatively smooth and flat in the IACL-R + a2MRS group compared to IACL-R group. Osteophyte was seen on both sides of the patellofemoral joint in the IACL-R group (Fig. 6B). Both MFC $(P<0.001)$ and MTP $(P<0.001)$ showed significant differences in CT values in the subchondral bone plate between the sham and IACL-R groups. In addition, significant differences in CT values were found between the IACL-R + a2MRS and IACL-R groups in the MFC $(P=0.028$; Fig. 6D). Significant differences in the thickness of the subchondral bone plate were found only between the sham and IACL-R groups in the MTP $(P=0.020$; Fig. 6E). MRI OSag-fs $P D$ showed that cartilage continuity was better without obvious local defects in the IACL-R + a2MRS group than in the IACL-R group (Fig. $6 C$, left). WORMS of the MFC $(P=0.006)$, MTP $(P=0.014)$ and sum $(P=0.004)$ were significantly lower in the IACL-R + a2MRS group than in the IACL-R group (Fig. 6G-I, Supplementary table 2). Osag T2MAP showed that regular orange-red layer were more obvious in the IACL-R + a2MRS group than in the IACL-R group (Fig. $6 \mathrm{C}$, right). The T2 values of the MFC $(P<0.001)$ and MTP $(P<0.001)$ were significantly lower in the IACL- + a2MRS group than in the IACL-R group (Fig. 6F).

Table 2 Kellgren-Lawrence grades on X-ray examination at 3 months $(n=6)$

\begin{tabular}{|lllllll|}
\hline Group & Grade & Grade & Grade & Grade & Grade & $\mathbf{P}$ \\
& $\mathbf{0}$ & $\mathbf{1}$ & $\mathbf{2}$ & $\mathbf{3}$ & $\mathbf{4}$ & \\
\hline sham & 3 & 2 & 1 & 0 & 0 & \\
\hline IACL-R & 0 & 1 & 3 & 2 & 0 & $>0.05$ \\
\hline IACL-R+a2M & 2 & 3 & 1 & 0 & 0 & \\
\hline
\end{tabular}

\section{Macroscopic Cartilage and Osteophyte Assessment}

Compared to that in the IACL-R group, cartilage degeneration was relatively low and no obvious cartilage defects or large erosions were found in the IACL-R + a2MRS group (Fig. 7A). OARSI scores of macroscopic cartilage were significantly lower in the IACL-R + a2MRS group than in the IACL-R group $(P=$ 0.031; Fig. 7B, Supplementary Fig. 1A-1E, Supplementary Table 2). Mild irregular protrusions were found only on sides of the trochlea in the IACL-R group (Fig. 7A). No differences were found in the OARSI sum scores of the osteophyte among the three groups $(P=0.438$; Figure $7 C$, Supplementary Figure 1F-1J, Supplementary Table 2).

\section{Histological Assessment}


In the IACLR + a2-MRS group compared with those in the IACLR-group, less decreases in safranin 0 staining and surface fibrillation were observed in the IACL-R + a2MRS group (Fig. 8A). The microscopic OARSI cartilage scores were lower in the IACL-R + a2MRS group than in the IACL-R group $(P=0.015$; Fig. 8B, Supplementary Fig. 2A-2E, Supplementary Table 2). Based on H\&E staining of the synovium, we found mild intimal thickening, low inflammatory cell infiltration, and slight sub-intimal fibrosis and vascularity in the IACL-R + a2MRS group, relative to those in the IACL-R group (Fig. 8C). Thus, the microscopic OARSI synovium scores, both total scores $(P=0.002)$ and single indicator scores, showed that synovial damage was lesser in the IACL-R + a2MRS group than in the IACL-R group (Fig. 8D, Supplementary Figure 2F-2I, Supplementary Table 2). H\&E staining of the meniscus also revealed mild surface fibrillation, normal cell distribution, and a normal collagen fiber organization (Fig. 8E), and the meniscus score was lower in the IACL-R + a2MRS group than in the IACL-R group $(P<0.001$; Fig. 8F,

\section{Supplementary Figure 2J-2L, Supplementary Table 2) .}

\section{Immunohistochemical Assessment}

Both articular cartilage (Fig. 9A-B) and synovium (Fig. 9C-D) immunostaining showed that MMP-3 (P $<0.001)$, MMP-9 $(P<0.001)$, MMP-13 ( $<<0.001)$, Col -10 ( $P<0.001)$, and Runx-2 $(P=0.001)$ staining significantly increased in the IACL-R group compared with the IACL-R + a2MRS group. In contrast, Col-2 (P $<0.001)$ expression in articular cartilage was higher in the IACL-R + a2MRS group than in the IACL- $R$ group.

\section{Discussion}

a2M performs complex bodily functions including the regulation of cytokine and hormone levels. It can bind several cytokines, including basic fibroblast growth factor, platelet-derived growth factor, nerve growth factor, IL-1 $\beta$, and IL-6, and regulate the levels of hepcidin and leptin[9]. The specific mechanism of a2M has been previously reported by Sottrup-Jensen [32]. Notably, recent studies showed that a2M can attenuate PTOA cartilage degeneration [15-17]. However, a2M is not present in SF at sufficient levels due to its large molecular weight, which prevents its migration from the blood into the SF to counteract the increased concentrations of catabolic factors that appear after joint injury[33]. Thus, introducing supplemental a2M in the joint cavity might be a strategy to attenuate cartilage degeneration. However, considering the expense and potential safety concerns of biosynthetic a2M, a2MRS is a promising alternative for PTOA treatment. The results of our study demonstrate, for the first time, that a2MRS, as a master inhibitor of inflammatory factors, can attenuate cartilage degeneration in vitro and in vivo.

First, our in vitro data clearly demonstrated that human a2MRS promotes the proliferation of human chondrocyte, reduces the apoptosis of these, and decreases chondrocyte catabolic cytokine gene transcription and secretion, suggesting that a2MRS is a promising therapy. The ultimate goal of studying a2MRS is clinical application. Thus, its biological safety and effectiveness need to be accurately evaluated and verified in vivo. 
Previous studies have demonstrated that that peak levels of cartilage catabolic enzymes could be detected on day 2 after joint injury [34]. Therefore, the mini pigs in the IACL-R + a2MRS group received the first a2MRS joint cavity injection 2 days after surgery, which inhibited the levels of various inflammatory factors. Luminex results revealed that not only were the peak concentrations of inflammatory factors in SF significantly reduced but the speed of decline was also faster in the IACL-R + a2MRS group than in the IACL-R group, which demonstrated the early effect of a2MRS. In addition, gait analysis showed that the gait ratios of left hind limb to right hind limb of the IACL-R + a2MRS group was significantly greater than 1 at 7 days post-surgery, but it was significantly lower than that in the IACL-R group, which further proves the effectiveness of a2MRS. Other research results ${ }^{37}$ suggest that the activity of some cartilage catabolic enzymes might have two peaks. The first phase appears after the initial trauma to the joint. The second peak is associated with progressive cartilage degeneration at weeks 4 and 6 after surgery. Therefore, we repeated a2MRS supplementation, which constantly exerts an inflammation-inhibitory effect, and the inflammatory storm or waterfall effect was interrupted in time[35]. Therefore, in the later stage (30-90 day) of the experiment, the concentration of inflammatory factors in the SF in the IACL-R + a2MRS group was maintained at a low level, and no obvious rebound was observed. Gait analysis is a relatively sensitive test for abnormal biomechanics and pain in the knee joint [36-38]. Compared with preoperative parameters, the gait of the IACL-R group also returned to previous levels, showing no significant difference in the middle stage (30-60 day). We concluded that the IACL-R model can restore the normal gait parameters of the knee joint and indirectly speculate that the ACL might have relatively stable biomechanical and consistent functions in different groups.

The mini pigs in the IACL-R + a2MRS group received a2MRS joint cavity injection four times starting from 2 days after surgery, which resulted in long-lasting inflammation suppression, significantly slowing the degeneration of articular cartilage. Therefore, the final imaging assessment, macroscopic cartilage assessment, and microscopic histological analysis confirmed that the articular cartilage in the IACL-R + a2MRS group was only slightly degenerated. Moreover, our biochemistry data demonstrated that supplemental a2MRS not only inhibited catabolic factors, including MMP-3, MMP-13, Col-10, and Runx-2, but also enhanced Col-2 and aggrecan gene expression and protein synthesis. The increase in collagen and aggrecan suggests that a2MRS might have cartilage-repair functions. This finding is consistent with previous reports [17]. Moreover, the results of H\&E staining of the synovium and meniscus proved that a2MRS injection into the joint cavity could significantly reduce inflammatory cell infiltration and vascularity, protecting the articular cartilage, synovium, and meniscus. In this study, a2MRS might act by binding cytokines in addition to directly neutralizing enzyme activities, but the exact mechanism is not clear. The relative contributions of these mechanisms will be addressed in future studies.

Our study has a few limitations. First, the state of tension in the ACL or biomechanical changes in the knee joint post-surgery are crucial. To date, there is no technology or instrument that can accurately detect the smaller motions between the femur and tibia that are controlled by the ACL. The gait analysis used in this study can only roughly or indirectly assess the biomechanical stability of the knee joint. Second, although the concentration of a2M in a2MRS was 6.48-fold higher than that in normal mini pig 
serum, a2MRS is still in fact a mixture containing extremely complex components. a2MRS was injected into the joint cavity, and proteins other than a2M might also play a role, but the exact mechanism is still unknown. Third, considering the side effects of the multiple anesthesia method used, we do not know the exact trend of inflammatory factor levels during the period from days 29 to 90 after surgery.

\section{Conclusion}

In summary, a2MRS is a promising bioinhibitor of catabolic proteases, and early and multiple injections in the joint cavity after IACL-R can significantly reduce the concentration of inflammatory factors in the joint SF of mini pigs and the degeneration of articular cartilage, exerting a chondroprotective effect.

\section{Abbreviations}

a2M: a2-Macroglobulin; a2MRS: a2M-rich serum; PTOA: post-traumatic osteoarthritis; ACL: Anterior cruciate ligament; ACL-R: ACL reconstruction; IACL-R: "idealized" anterior cruciate ligament autograft reconstruction;SF :synovial fluid;ELISA: Enzyme-Linked ImmunoSorbent Assay;IL: interleukin ;MMP13:matrix metalloproteinase 13; TNF-a:tumor necrosis factor-a ;CCK-8:Cell Counting Kit-8 ;TUNEL :Terminal transferase dUTP end Labeling; GM-CSF $₫$ granulocyte-macrophage colony-stimulating factor》 GAG, glycosaminoglycan ; CT:computed tomography; 3D CT: three-dimensional CT reconstruction; MRI :magnetic resonance imaging ; WORMS :whole-organ MRI score ; MFC:medial femoral condyle; LFC:Iateral femoral condyle; MTP: medial tibial plateau; LTP:Iateral tibial plateau;OARSI :Osteoarthritis Research Society International (OARSI);H\&E :hematoxylin and eosin ; LH: left hind foot; RH: right hind foot

\section{Declarations}

\section{Ethics approval and consent to participate:}

All procedures in this study were approved by the Ethics Committee of the Second Hospital of Shanxi Medical University ( NO. SYDL2019001).

\section{Consent for publication:}

Not applicable

\section{Availability of data and materials:}

The datasets used and or analysed during the current study are available from the corresponding author on reasonable request

\section{Competing interests:}

The authors declare that they have no competing interests 


\section{Funding:}

The project was funded by the International Science and Technology Cooperation Program of China [Grant No. 2015DFA33050]

\section{Authors' contributions:}

RPZ,LAHparticipated in the study design, wrote the manuscript, performed most of the experiments, and analyzed data.XCW,LAH conceived the study, revised the manuscript.CMZ,HRW,CX,WPD,ZQD carried out the part of imaging and histological assessment and helped to perform the statistical analysi.WPD,HQL, CJL.YZ,performed the IACL-R of minipigs and gait Assessment .All authors read and approved the final manuscript.

\section{Acknowledgments}

We would like to thank Yi Xu and Feipeng Song for providing assistance with the imaging examination, and Yipeng Xue, a veterinarian at the Sciences of ShanXi Agricultural Academy Institute of Animal Husbandry \& Veterinary, for teaching us aboutanimal management. Besides $₫$ We would like to thank Pengfei Han (Heping Hospital Affiliated to Changzhi Medical College), Xiaodong Gu (Bethune Hospital, Shanxi Medical University) and Pengcui Li( The Second Hospital of Shanxi Medical University) for providing assistance with the minipig model of IACL-R..

\section{References}

1. Barenius B, Sari P, Adel S, Robert B, Louise N, Karl E: Increased risk of osteoarthritis after anterior cruciate ligament reconstruction: a 14-year follow-up study of a randomized controlled trial. Am J Sports Med 2014, 42(5):1049-1057.

2. Björnsson H, Samuelsson K, Sundemo D, Desai N, Sernert N, Rostgård-Christensen L, Karlsson J, Kartus J: A Randomized Controlled Trial With Mean 16-Year Follow-up Comparing Hamstring and Patellar Tendon Autografts in Anterior Cruciate Ligament Reconstruction. American Journal of Sports Medicine 2016, 44(9): 2304-13.

3. Wang LJ, Zeng N, Yan ZP, Li JT, Ni GX: Post-traumatic osteoarthritis following ACL injury. Arthritis Res Ther 2020, 22(1):57.

4. Bryan J. Heard YA, 1 May Chung,1 Nigel G. Shrive,1,2 Cyril B. Frank1: Early Joint Tissue Changes Are Highly Correlated with a Set of Inflammatory and Degradative Synovial Biomarkers after ACL Autograft and Its Sham Surgery in an Ovine Model. J Orthop Res.. 2011;29:1185-92.

5. Han PF, Wei L, Duan ZQ, Zhang ZL, Chen TY, Lu JG, Zhao RP, Cao XM, Li PC, Lv Z et al: Contribution of IL-1beta, 6 and TNF-alpha to the form of post-traumatic osteoarthritis induced by "idealized" anterior cruciate ligament reconstruction in a porcine model. Int Immunopharmacol 2018, 65:212220. 
6. Heard BJ, Solbak NM, Chung M, Achari Y, Shrive NG, Frank CB, Hart DA: The infrapatellar fat pad is affected by injury induced inflammation in the rabbit knee: use of dexamethasone to mitigate damage. Inflammation research: official journal of the European Histamine Research Society [et al] 2016, 65(6):459-470.

7. Lieberthal J, Sambamurthy N, Scanzello CR: Inflammation in joint injury and post-traumatic osteoarthritis. Osteoarthritis Cartilage 2015, 23(11):1825-1834.

8. Maerz T, Sherman E, Newton M, Yilmaz A, Kumar P, Graham SF, Baker KC: Metabolomic serum profiling after $A C L$ injury in rats: A pilot study implicating inflammation and immune dysregulation in post-traumatic osteoarthritis. J Orthop Res 2018, 36(7):1969-1979.

9. Rehman AA, Ahsan H, Khan FH: alpha-2-Macroglobulin: a physiological guardian. J Cell Physiol 2013, 228(8):1665-1675.

10. Ho AS, Cheng CC, Lee SC, Liu ML, Lee JY, Wang WM, Wang CC: Novel biomarkers predict liver fibrosis in hepatitis $C$ patients: alpha 2 macroglobulin, vitamin $D$ binding protein and apolipoprotein $A I$. Journal of biomedical science 2010, 17(1):58.

11. Chung TJ, Hsu KY, Chen JH, Liu JS, Chang HW, Li PF, Huang CL, Shieh YS, Lee CH: Association of salivary alpha 2-macroglobulin levels and clinical characteristics in type 2 diabetes. $J$ Diabetes Investig 2016, 7(2):190-196.

12. Cuellar JM, Cuellar VG, Scuderi GJ: alpha2-Macroglobulin: Autologous Protease Inhibition Technology. Phys Med Rehabil Clin N Am 2016, 27(4):909-918.

13. Montesano G, Cuellar J: Improving response to treatment for patients with DDD by the use of molecular markers. 2015. 4th International Conference on Orthopedics \& Rheumatology. October 2628, 2015 Baltimore, Maryland, USA

14. Li J, Kong XB, Chen XY, Zhong WZ, Chen JY, Liu Y, Yin P, Fang SL: Protective role of alpha2macroglobulin against jaw osteoradionecrosis in a preclinical rat model. J Oral Pathol Med 2019, 48(2):166-173.

15. Li S, Xiang C, Wei X, Sun X, Li R, Li P, Sun J, Wei D, Chen Y, Zhang Y et al: Early supplemental a2macroglobulin attenuates cartilage and bone damage by inhibiting inflammation in collagen IIinduced arthritis model. Int J Rheum Dis 2019, 22(4):654-665.

16. Wang S, Wei X, Zhou J, Zhang J, Li K, Chen Q, Terek R, Fleming BC, Goldring MB, Ehrlich MG: Identification of a2-macroglobulin as a master inhibitor of cartilage-degrading factors that attenuates the progression of posttraumatic osteoarthritis. Arthritis \& Rheumatology 2014, 66(7):1843-1853.

17. Zhang Y, Wei X, Browning S, Scuderi G, Hanna LS, Wei L: Targeted designed variants of alpha-2macroglobulin (A2M) attenuate cartilage degeneration in a rat model of osteoarthritis induced by anterior cruciate ligament transection. Arthritis Res Ther 2017, 19(1):175.

18. Guo L, Wei X, Zhang Z, Wang X, Wang C, Li P, Wang C, Wei L: Ipriflavone attenuates the degeneration of cartilage by blocking the Indian hedgehog pathway. Arthritis Res Ther 2019, 21(1):109. 
19. Gu XD, Wei L, Li PC, Che XD, Zhao RP, Han PF, Lu JG, Wei XC: Adenovirus-mediated transduction with Histone Deacetylase 4 ameliorates disease progression in an osteoarthritis rat model. Int Immunopharmacol 2019, 75:105752.

20. Wei L, Fleming BC, Sun X, Teeple E, Wu W, Jay GD, Elsaid KA, Luo J, Machan JT, Chen Q: Comparison of differential biomarkers of osteoarthritis with and without posttraumatic injury in the Hartley guinea pig model. J Orthop Res 2010, 28(7):900-906.

21. Shah KC, Linsley CS, Wu BM: Evaluation of a shape memory implant abutment system: An up to 6month pilot clinical study. The Journal of prosthetic dentistry 2020, 123(2):257-263.

22. Rashid MH, Theberge Y, Elmes SJ, Perkins MN, Mcintosh F: Pharmacological validation of early and late phase of rat mono-iodoacetate model using the Tekscan system. European Journal of Pain $2013,17(2): 210-222$.

23. Ruan MZC, Patel RM, Dawson BC, Jiang MM, Lee BHL: Pain, motor and gait assessment of murine osteoarthritis in a cruciate ligament transection model. Osteoarthritis Cartilage 2013, 21(9):13551364.

24. Misir A, Yildiz KI, Kizkapan TB, Incesoy MA: Kellgren-Lawrence grade of osteoarthritis is associated with change in certain morphological parameters. The Knee 2020, 27(3):633-641.

25. Kellgren JH, Lawrence JS: Radiological Assessment of Osteo-Arthrosis. Annals of the Rheumatic Diseases 1957, 16(4):494-502.

26. Hu W, Chen Y, Dou C, Dong S: Microenvironment in subchondral bone: predominant regulator for the treatment of osteoarthritis. Ann Rheum Dis 2020, 80(4):413-422.

27. Xue Y, Chen Y, Jiang D, Wang L, Wang X, Li M, Wu Y, Zhang M, Pang J, Zhan H et al: Self-Reported Weather Sensitivity is Associated with Clinical Symptoms and Structural Abnormalities in Patients with Knee Osteoarthritis: A Cross-Sectional Study. Rheumatology and therapy 2021, 8(3):14051417.

28. Pozzi F, Snyder-Mackler L, Zeni J, Jr.: Relationship between biomechanical asymmetries during a step up and over task and stair climbing after total knee arthroplasty. Clin Biomech (Bristol, Avon) 2015, 30(1):78-85.

29. Little CB, Smith MM, Cake MA, Read RA, Murphy MJ, Barry FP: The OARSI histopathology initiative recommendations for histological assessments of osteoarthritis in sheep and goats. Osteoarthritis Cartilage 2010, 18 Suppl 3:S80-92.

30. Pauli C, Grogan SP, Patil S, Otsuki S, Hasegawa A, Koziol J, Lotz MK, D'Lima DD: Macroscopic and histopathologic analysis of human knee menisci in aging and osteoarthritis. Osteoarthritis Cartilage 2011, 19(9):1132-1141.

31. Li L, Wei X, Wang D, Lv Z, Geng X, Li P, Lu J, Wang K, Wang X, Sun J et al: Positive Effects of a Young Systemic Environment and High Growth Differentiation Factor 11 Levels on Chondrocyte Proliferation and Cartilage Matrix Synthesis in Old Mice. Arthritis Rheumatol2020, 72(7):1123-1133.

32. Sottrup-Jensen L: Alpha-macroglobulins: structure, shape, and mechanism of proteinase complex formation. J Biol Chem 1989, 264(20):11539-11542. 
33. Salvesen G, Enghild JJ: alpha-Macroglobulins: detection and characterization. Methods in enzymology 1993, 223:121-141.

34. Zhao R, Dong Z, Wei X, Gu X, Han P, Wu H, Yan Y, Huang L, Li H, Zhang C et al: Inflammatory factors are crucial for the pathogenesis of post-traumatic osteoarthritis confirmed by a novel porcine model: "Idealized" anterior cruciate ligament reconstruction" and gait analysis. Int Immunopharmaco/2021, 99:107905.

35. Pan LP, Cao YP, Wen LC, Chai WB, Jun-Bao DU, Jin HF, Liu J, Yang X, Meng ZC, Liu H: Hydrogen sulfide in cartilage and its inhibitory effect on matrix metalloproteinase 13 expression in chondrocytes induced by interlukin-1 $\beta$. Journal of Peking University 2016.

36. Muramatsu Y, Sasho T, Saito M, Yamaguchi S, Akagi R, Mukoyama S, Akatsu Y, Katsuragi J, Fukawa $\mathrm{T}$, Endo $\mathrm{J}$ et al: Preventive effects of hyaluronan from deterioration of gait parameters in surgically induced mice osteoarthritic knee model. Osteoarthritis Cartilage 2014, 22(6):831-835.

37. Capin JJ, Khandha A, Zarzycki R, Manal K, Buchanan TS, Snyder-Mackler L: Gait Mechanics After ACL Reconstruction Differ According to Medial Meniscal Treatment. J Bone Joint Surg Am 2018, 100(14):1209-1216.

38. Hughes-Oliver CN, Srinivasan D, Schmitt D, Queen RM: Gender and limb differences in temporal gait parameters and gait variability in ankle osteoarthritis. Gait Posture 2018, 65:228-233.

\section{Figures}




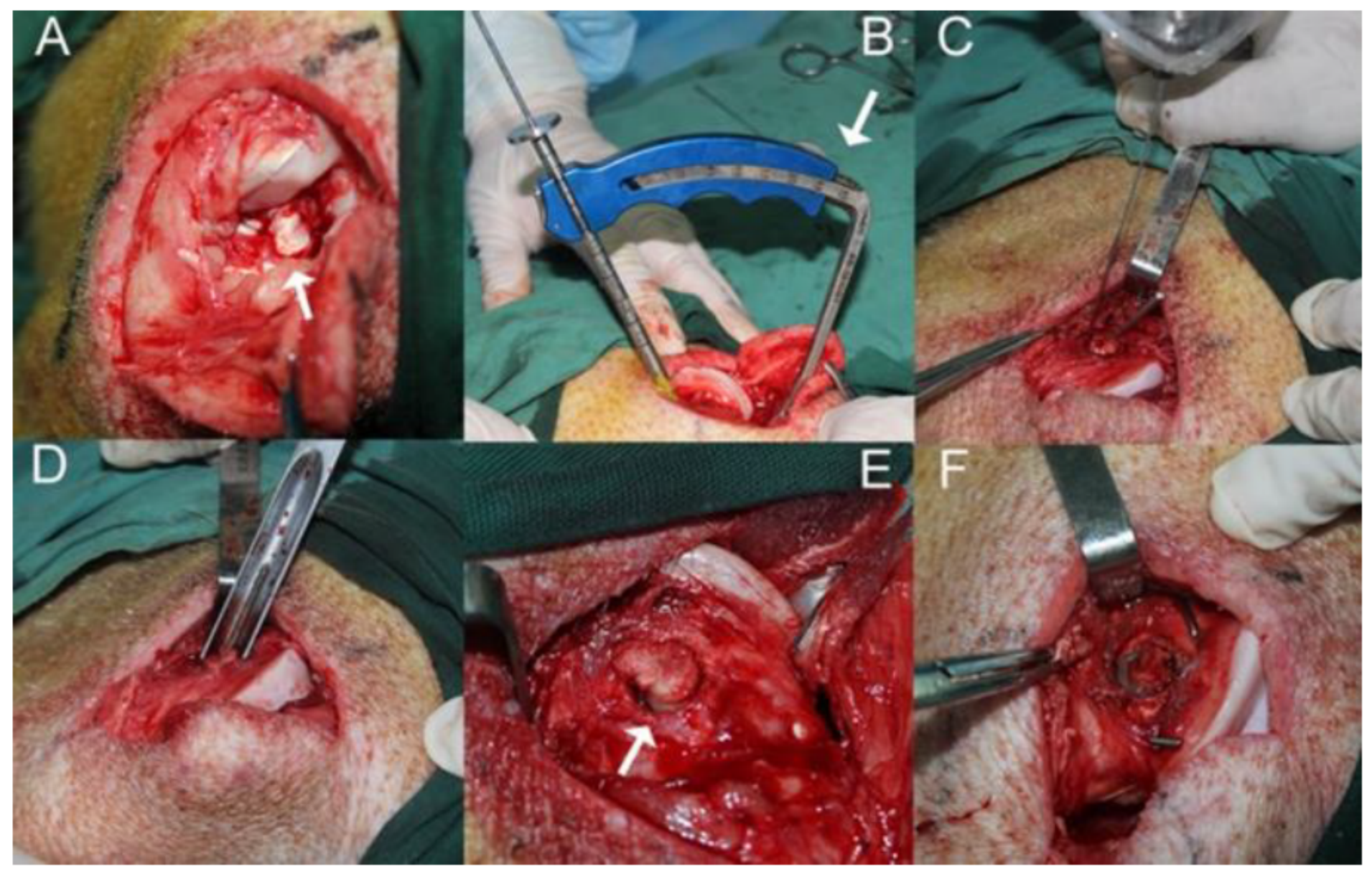

\section{Figure 1}

The surgical procedure performed on the right hind limb of a minipig in the IACL-R and IACL-R+a2MRS groups. A: The stifle joint was open and the patella was dislocated to expose the ACL (arrow). B: The ACL reconstruction guide was positioned at a $45^{\circ}$ angle (arrow) to the longitudinal axis of the femur. C: Before the hollow drill was about to penetrate the femoral tunnel, a Kirschner wire (diameter $1 \mathrm{~mm}$ ) was drilled along outer edge of the tunnel to prevent the cartilage from splitting. D: The tunnel was gently penetrated by the same diameter thin-walled ring osteotomy to completely severv tendon-bone segment $\mathrm{E}$ : The tendon-bone segment was completely freed (arrow). F: The tendon-bone segment was fixed in situ with two crossed Kirschner wires. 

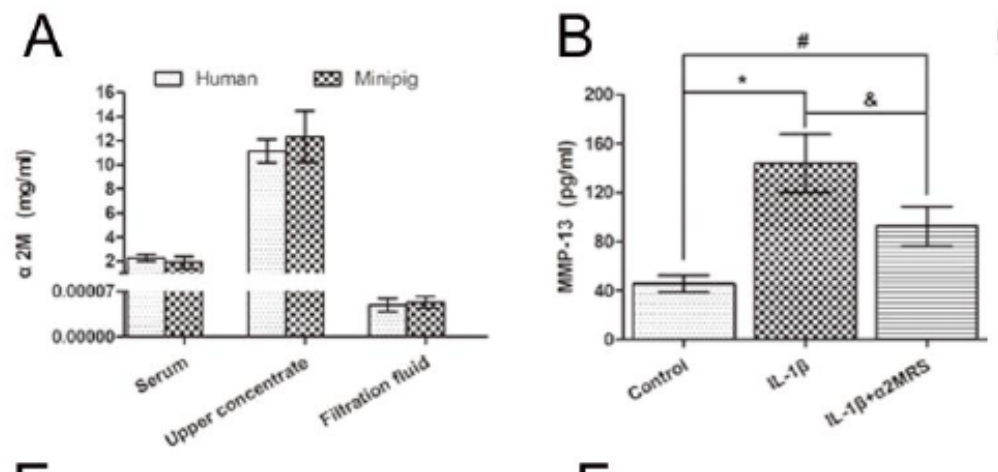

F TUNEL

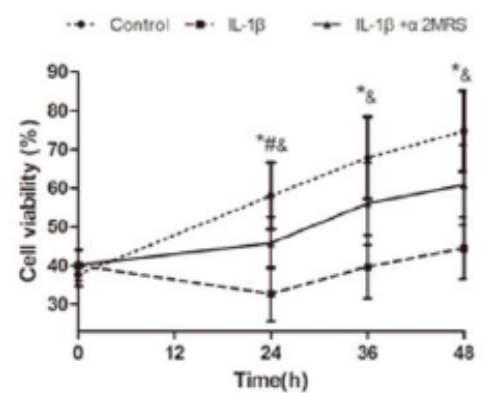

G

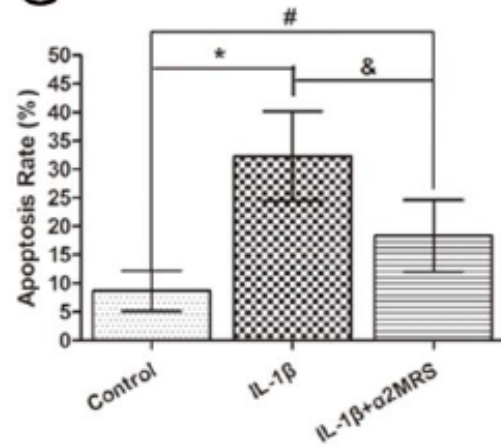

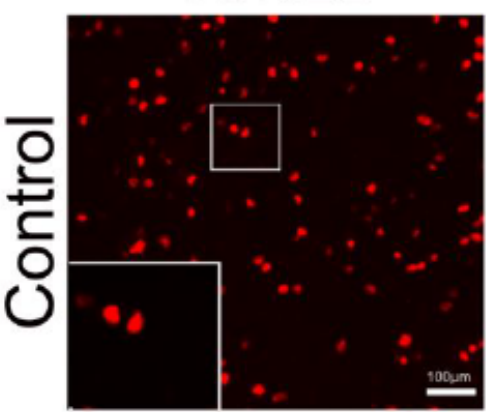
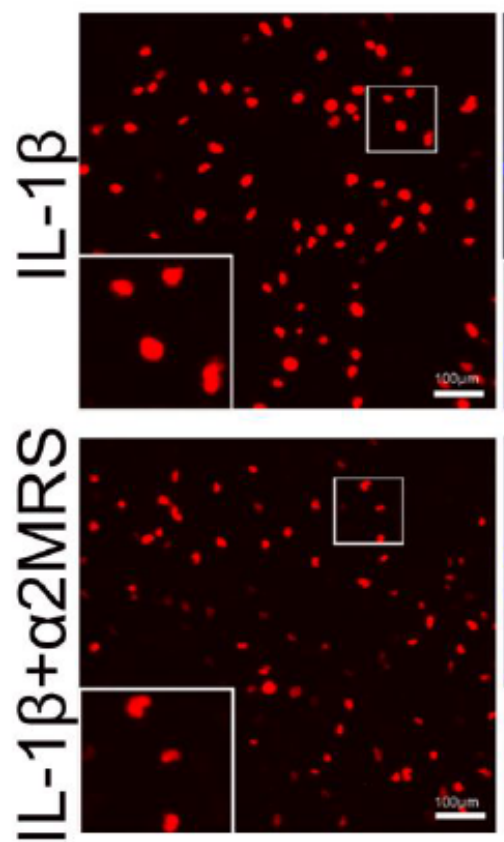

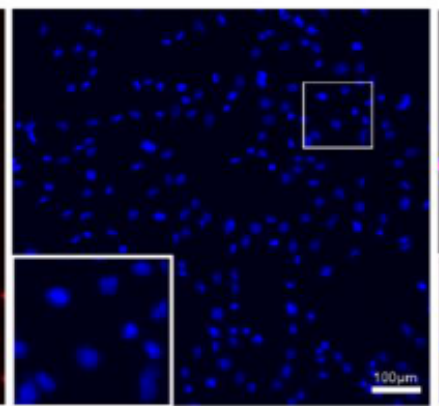

C

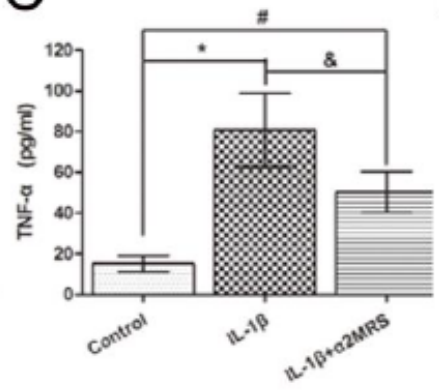

DAPI
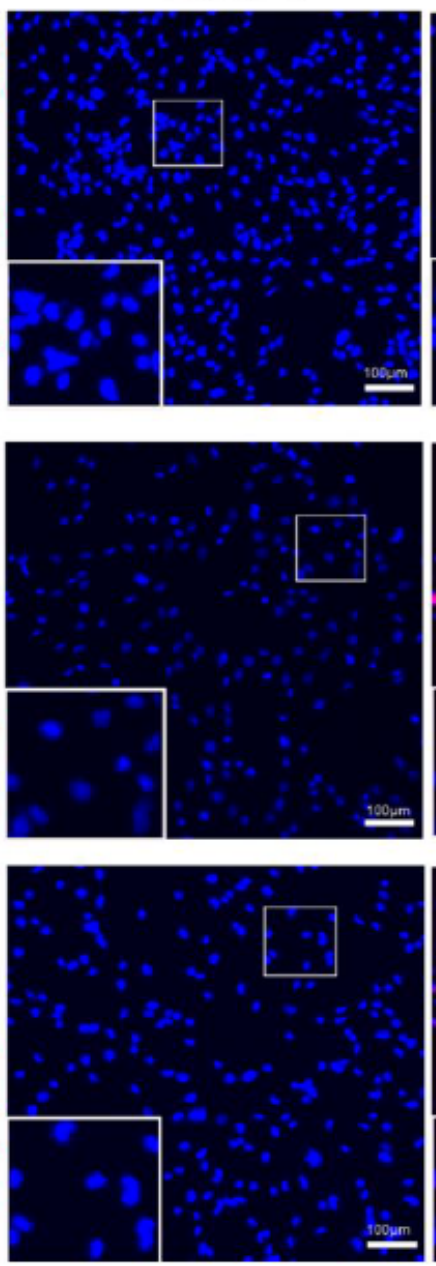

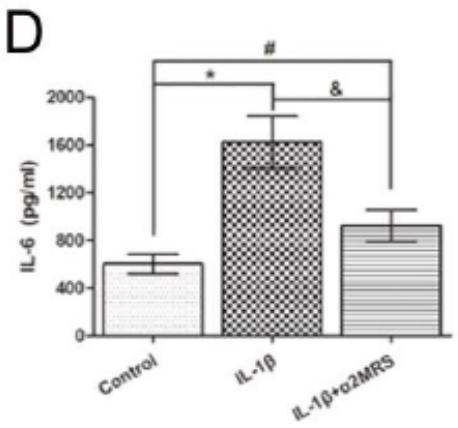

Merge
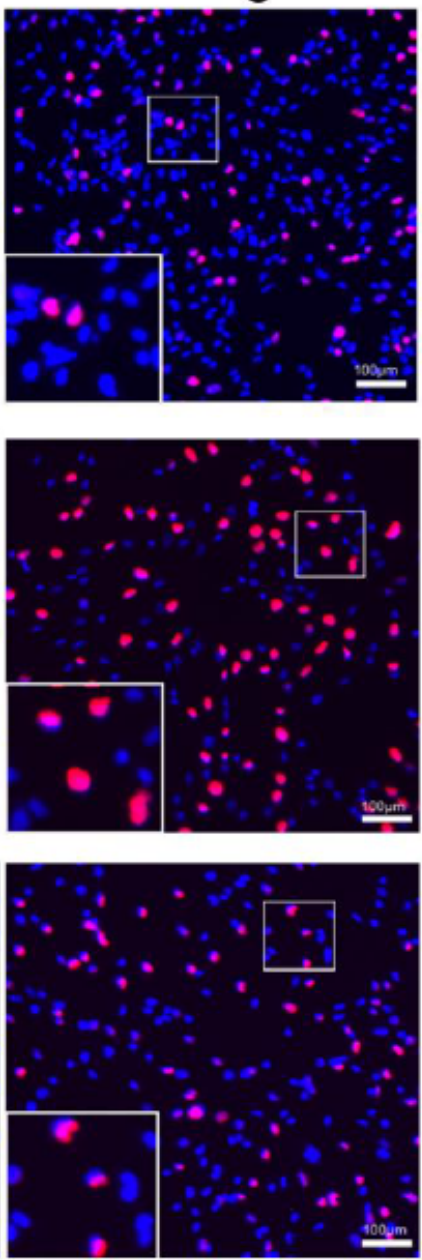

\section{Figure 2}

A. Human and mini pig a2M concentrations after centrifugation at $5000 \times \mathrm{g}$ for $30 \mathrm{~min}$. Human: serum: $2.28 \pm 0.26 \mathrm{mg} / \mathrm{ml}$; upper concentrate: $11.13 \pm 0.90 \mathrm{mg} / \mathrm{ml}$; filtration fluid: $0.000049 \pm 0.00001 \mathrm{mg} / \mathrm{ml}$; mini pigs: serum: $1.90 \pm 0.46 \mathrm{mg} / \mathrm{ml}$; upper concentrate: $12.32 \pm 1.97 \mathrm{mg} / \mathrm{ml}$; filtration fluid: $0.000053 \pm 0.000008$ $\mathrm{mg} / \mathrm{ml}$. B-D. a2MRS significantly inhibited the induction of MMP-13,TNF- $\alpha$ and IL- 6 in IL-1 $\beta$ induced human primary osteoarthritic chondrocytes. E: The CCK-8 assay results showed that the viability of chondrocytes was higher in IL-1 $\beta+\alpha 2 M R S$ group relative to that in the IL-1 $\beta$ group, and the viability gradually increased with a longer treatment time. F:TUNEL assay results showed that apoptosis (red) was reduced in the IL-1 $\beta+\alpha 2 M R S$ group seem to that in the IL-1 $\beta$ group. The bottom panels are higher- 
magnification views of the boxed areas in the top panels G: The percentage of TUNEL-positive cells was quantified, and the apoptosis rate of chondrocytes was significantly reduced in the IL-1 $\beta+\alpha 2 M R S$ group relative to that in the IL-1 $\beta$ group. ${ }^{*}=P<0.05$, control group versus IL-1 $\beta$ group; $\#=P<0.05$, control group versus IL-1 $\beta+\alpha 2 M R S$ group; $\&=P<0.05$, IL-1 $\beta$ group versus IL-1 $\beta+\alpha 2 M R S$ group. The bars show the mean $\pm \operatorname{SD}(n=6)$.
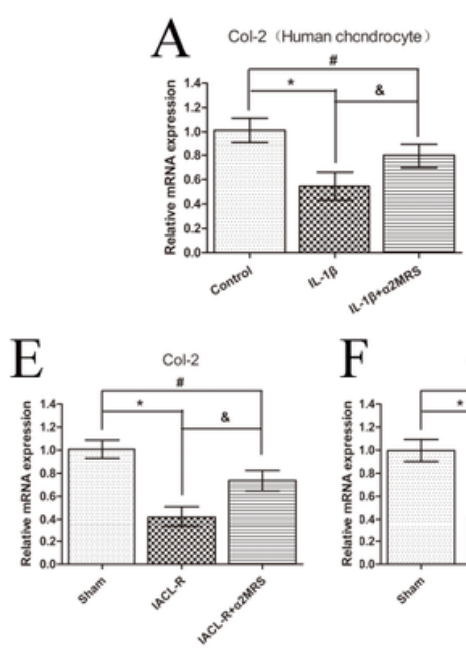
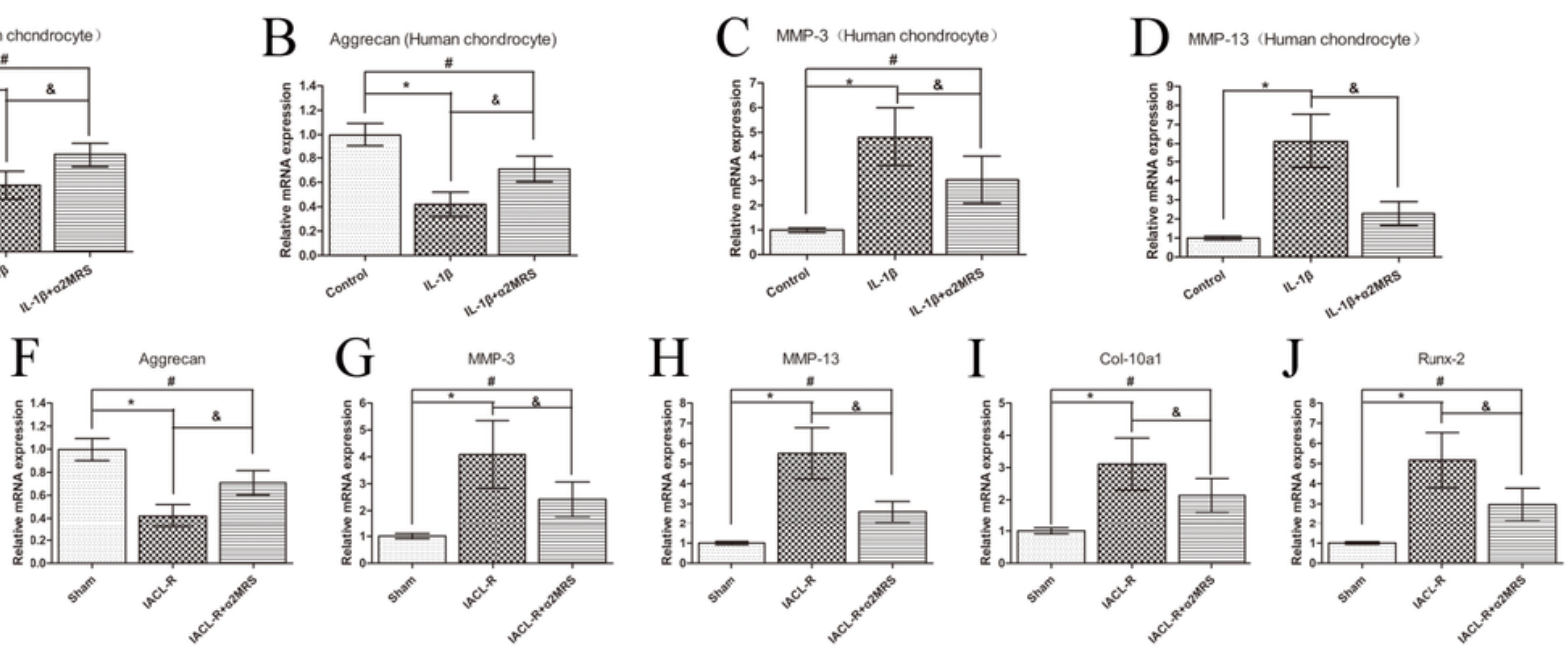

\section{Figure 3}

Real-time PCR data indicated that supplementation with a2MRS reduced cartilage matrix catabolism and enhanced anabolism in vitro $(A-D)$ and in vivo $(E-G)$. mRNA levels of MMP-3 $(P<0.001), M M P-13(P<$ 0.001), Col-10 a1 ( $P=0.001)$, and Runx-2 $(P<0.001)$ were lower in IL-1 $\beta+\alpha 2 M R S$ and IACL-R+a2MRS groups than in IL-1 $\beta$ and IACL-R groups respectively. In contrast, mRNA levels of Col-2 $(P<0.001)$ and aggrecan $(P<0.001)$ followed the opposite pattern. Both were increased in IL-1 $\beta+a 2 M R S$ and IACL$\mathrm{R}+\mathrm{a} 2 \mathrm{MRS}$ groups as compared to levels in IL- $1 \beta$ and IACL-R groups. ${ }^{*}=\mathrm{P}<0.05$, control group versus IL$1 \beta$ group or sham group versus IACL-R group; $\#=P<0.05$, control group versus IL- $1 \beta+a 2 M R S$ group or sham group versus IACL-R+a2MRS group; $\&=P<0.05$, IL-1 $\beta$ group versus IL- $1 \beta+\alpha 2 M R S$ group or IACL- $R$ group versus IACL-R+a2MRS group. The bars show the mean $\pm S D(n=6)$. 

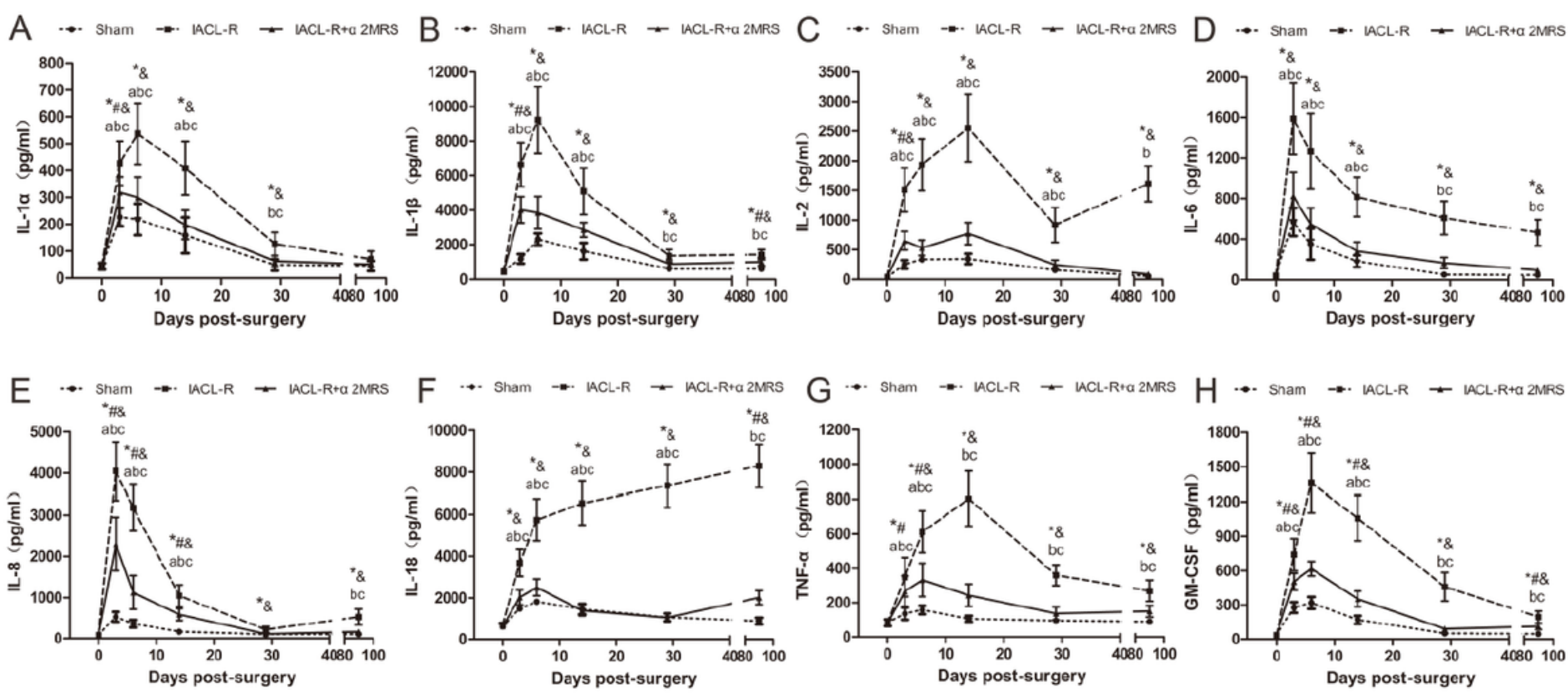

\section{Figure 4}

Line charts of inflammatory factors in synovial fluid.A: IL-1a; B: IL-13; C: IL-2; D: IL-6; E: IL-8; F: IL-18; G: TNF-a; H: GM-CSF *=P<0.05, sham group versus IACL-R group at the same time point; \#=P<0.05, sham group versus IACL-R+a2MRS group at the same time point; \&=P $<0.05$, IACL-R group versus IACL$\mathrm{R}+\mathrm{a} 2 \mathrm{MRS}$ group at the same time point. $\mathrm{a}=\mathrm{P}<0.05$, preoperative (day 0 ) versus postoperative (days 3,6 , 14,29 , and 90 ) in the sham group; $b=P<0.05$, preoperative (day 0 ) versus postoperative (days $3,6,14,29$, and 90 ) in the IACL-R group; $C=P<0.05$, preoperative (day 0 ) versus postoperative (days $3,6,14,29$, and $90)$ in the IACL-R+a2MRS group. The bars show the mean $\pm S D(n=6)$
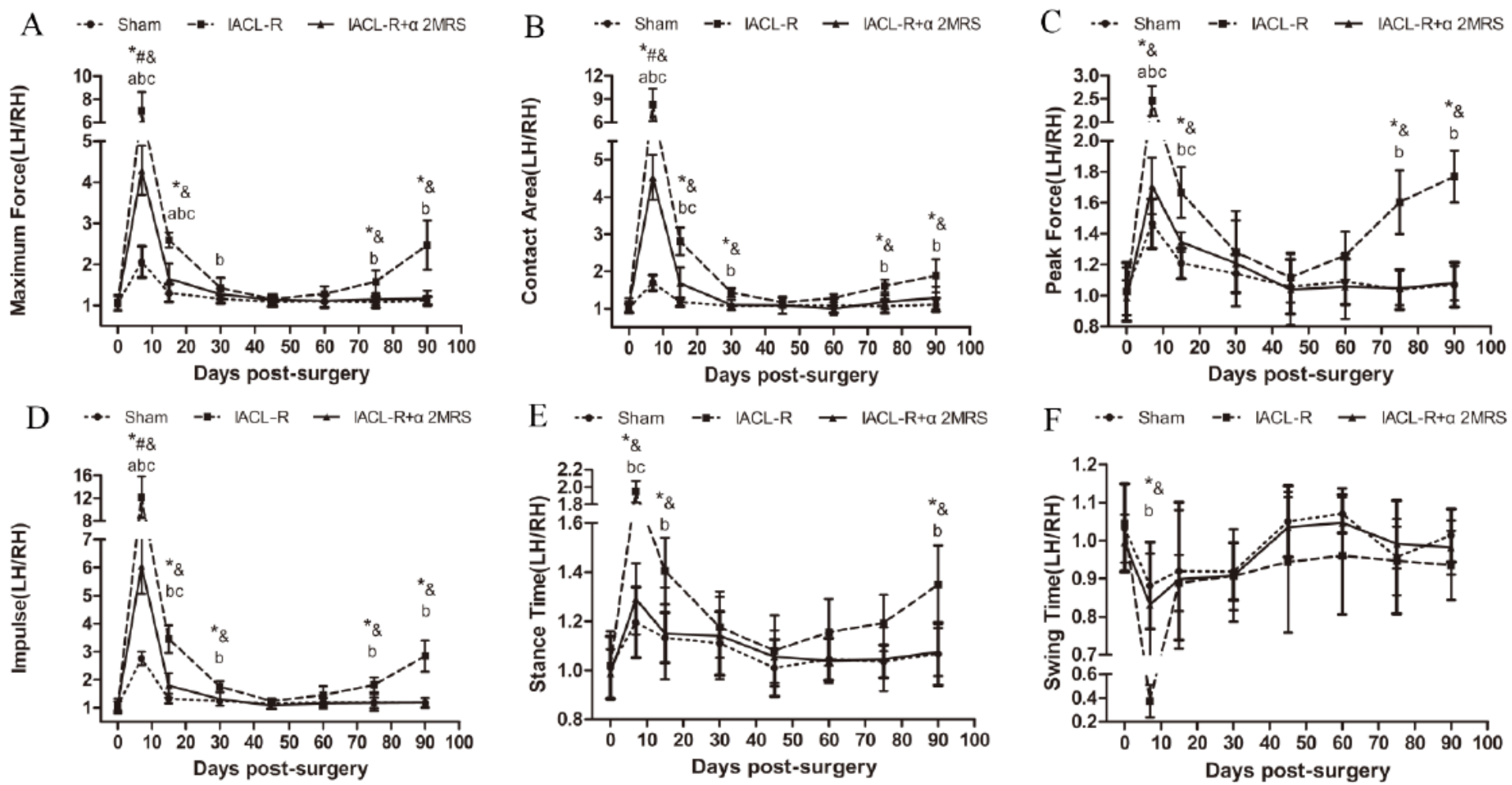


\section{Figure 5}

Line charts of gait analysis. A:Maximum force; B:Contact area; C:Peak force; D:Impulse; E:Stance time; $F$ :Swing time ${ }^{*}=P<0.05$, sham group versus IACL-R group at the same time point; $\#=P<0.05$, sham group versus IACL-R+a2MRS group at the same time point; \&=P $<0.05$, IACL-R group versus IACL$\mathrm{R}+\mathrm{a} 2 \mathrm{MRS}$ group at the same time point. $\mathrm{a}=\mathrm{P}<0.05$, preoperative (day 0 ) versus postoperative (days 7 , $15,30,45,60,75$, and 90 ) in the sham group; $b=P<0.05$, preoperative (day 0 ) versus postoperative (days $7,15,30,45,60,75$, and 90 ) in the IACL-R group; $c=P<0.05$, preoperative (day 0 ) versus postoperative (days $7,15,30,45,60,75$, and 90$)$ in the IACL-R+a2MRS group. The bars show the mean $\pm S D(n=6)$ 


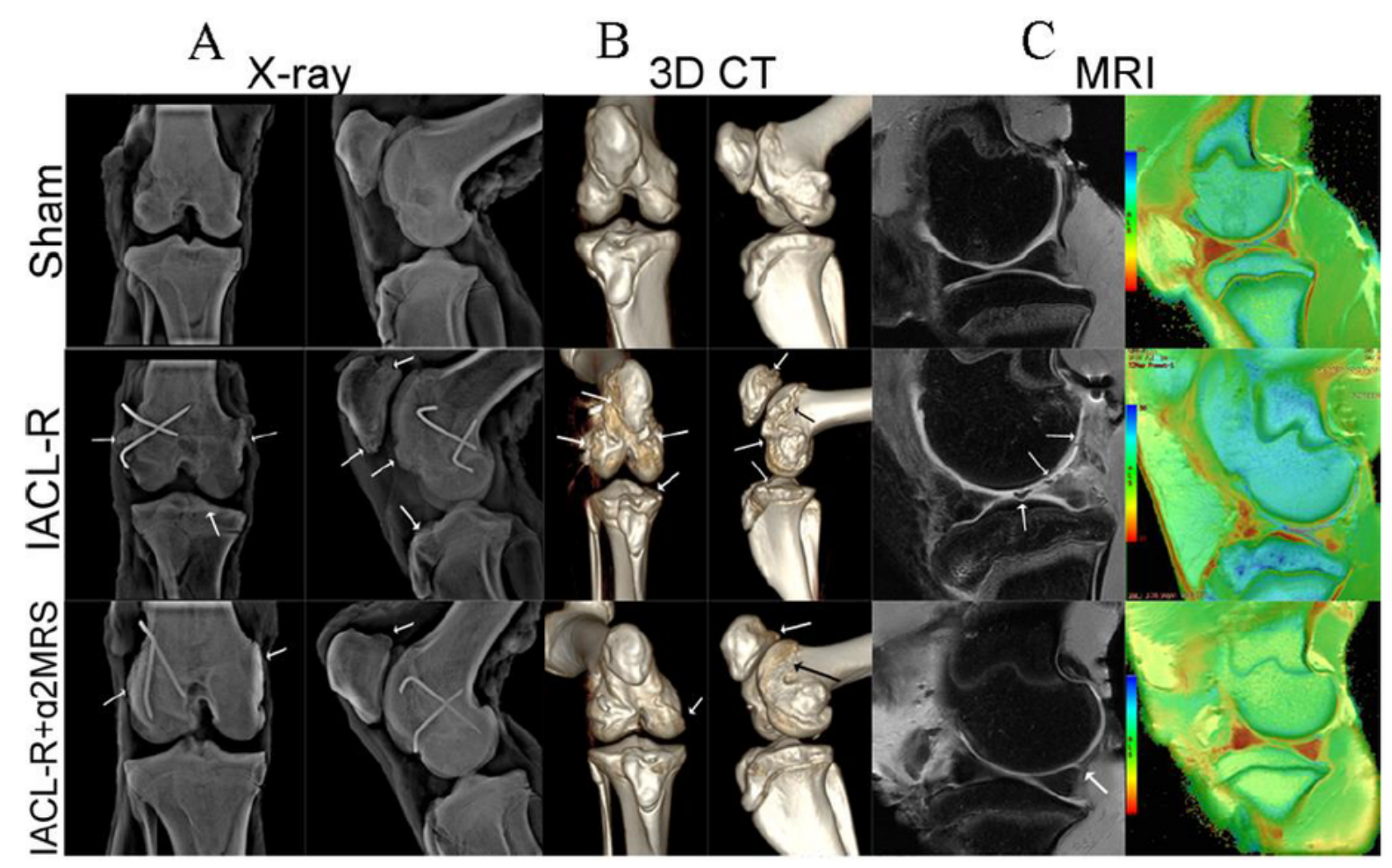

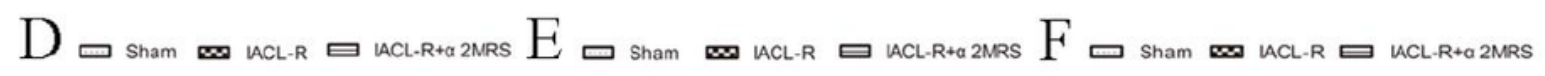
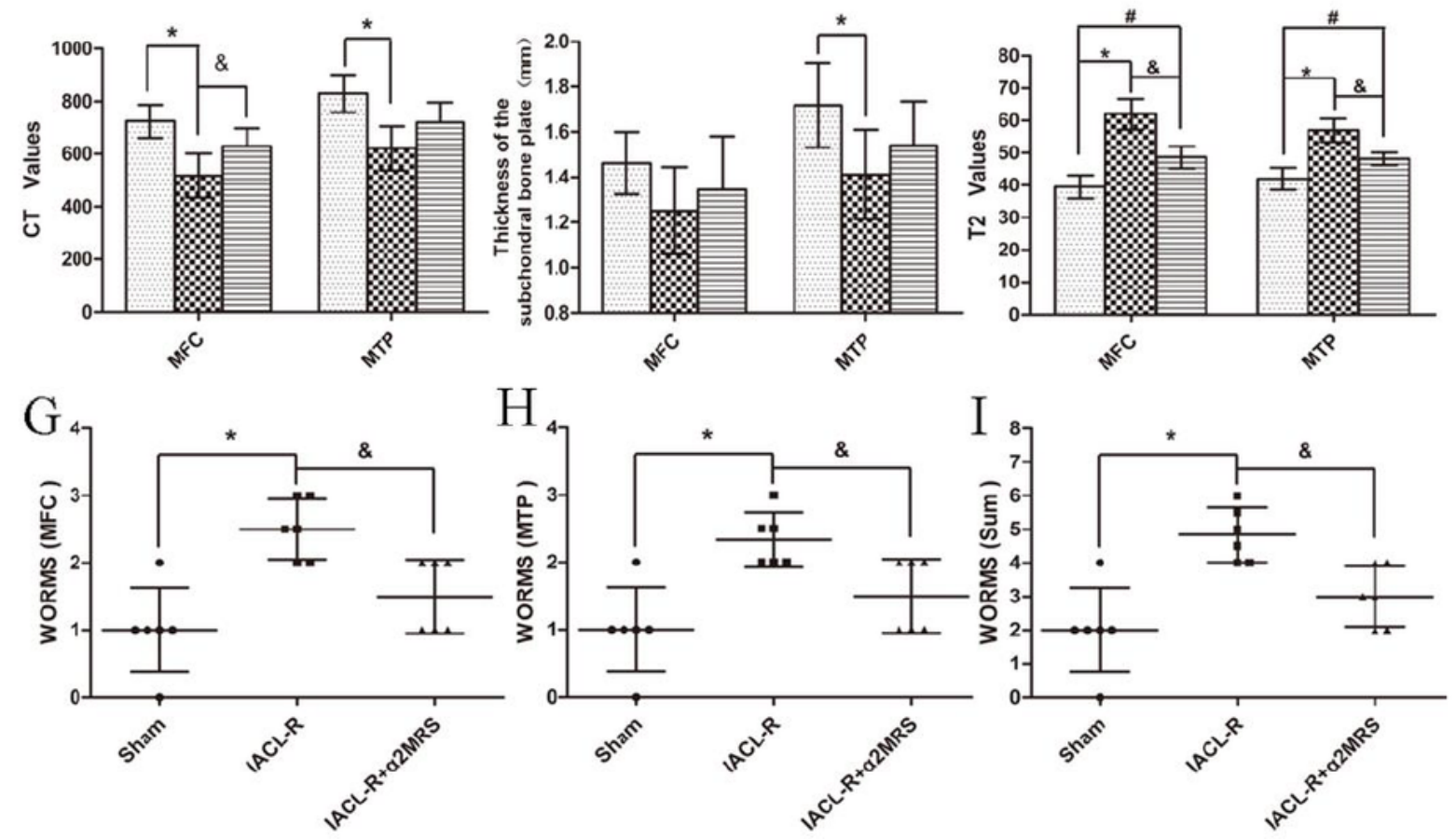

\section{Figure 6}

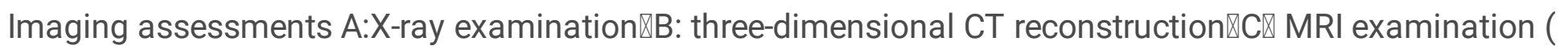

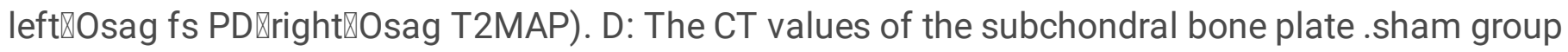
(MFC,724.2 \pm 62.95; MTP, 830.3 \pm 71.26 );IACL-R group (MFC, 517.7 \pm 83.89; MTP, 621.2 \pm 83.57); IACL$R+a 2 M R S$ group(MFC, $630 \pm 66.9 ;$ MTP, $719.2 \pm 76.73)$. E: The thickness of the subchondral bone plate. sham group (MFC, 1.46 0.14; MTP, $1.72 \pm 0.19) ;$;ACL-R group (MFC, $1.25 \pm 0.19 ;$ MTP, $1.41 \pm 0.20)$; IACL- 
R+a2MRS group(MFC, $1.35 \pm 0.24 ;$ MTP, $1.54 \pm 0.19)$. F: Quantification of the T2 values obtained using MRI sag T2MAP.Sham group( MFC, $39.5 \pm 3.62$; MTP, $42 \pm 3.41$ ); IACL-R group( MFC, $62 \pm 4.78$; MTP, $57 \pm$ 3.58); IACL-R+a2MRS group(MFC, $48.67 \pm 3.45$; MTP, $48.17 \pm 1.94)$. G-I: Quantification of the MRI OSag fs PD results using the WORMS guidelines. MFC, medial femoral condyle; MTP, medial tibial plateau. The white arrows indicate irregularities, osteophytes, and cartilage defects. ${ }^{*}=\mathrm{P}<0.05$, sham group versus IACL-R group; \#=P < 0.05, sham group versus IACL-R+a2MRS group; $\&=P<0.05$, IACL-R group versus IACL-R+a2MRS group. The bars show the mean $\pm S D(n=6)$.
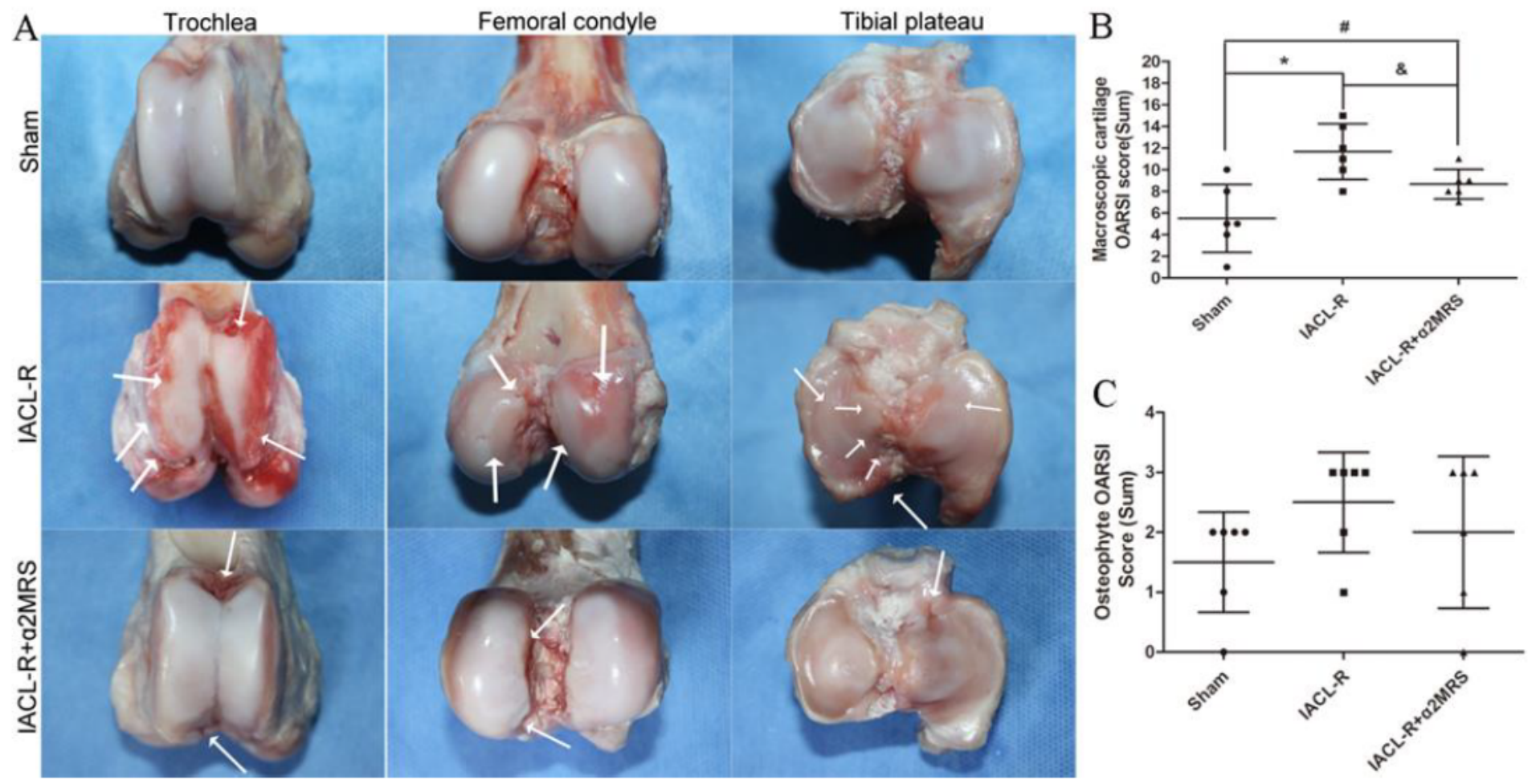

\section{Figure 7}

A: Images of the trochlea, femoral condyle, and tibial plateau for the macroscopic cartilage and osteophyte assessment according to OARSI guidelines. The white arrows indicate cartilage damage, irregularities, and osteophytes. B : Macroscopic cartilage score were lower in the IACL-R+a2MRS group than in the IACL-R group $(P=0.031)$. C: Macroscopic osteophyte scores did not differ among the different groups $(P=0.438) .{ }^{*}=P<0.05$, sham group versus IACL-R group; $\#=P<0.05$, sham group versus IACL$R+a 2 M R S$ group; $\&=P<0.05$, IACL-R group versus IACL-R+a2MRS group. The bars show the mean $\pm S D$ $(n=6)$. 
A cartilage (Safo/FG) C Cartilage (Safo/FG)

C Synovium (H\&E)
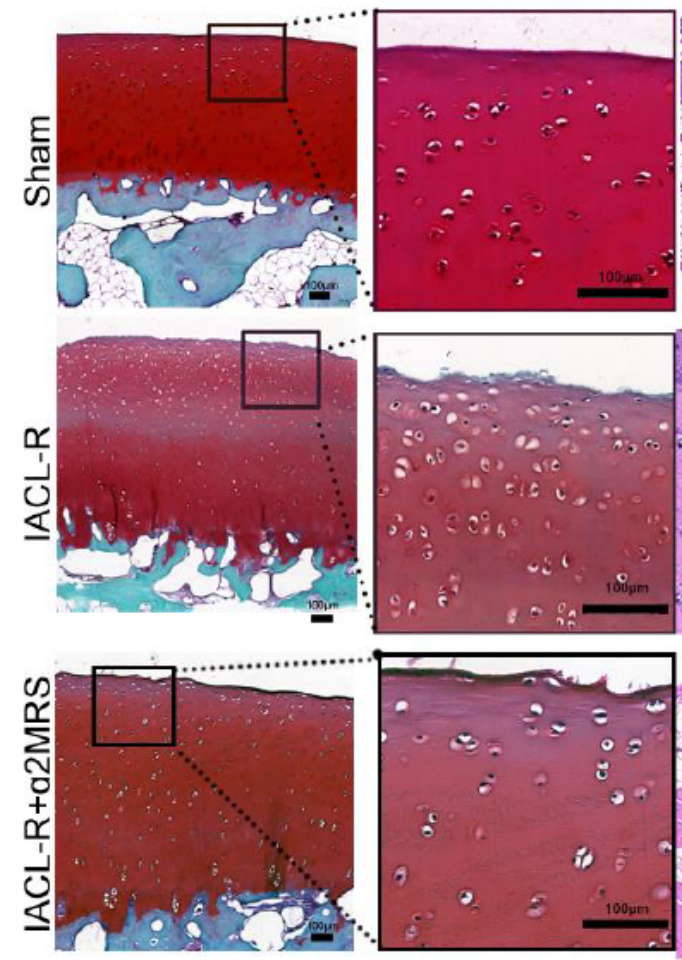

B

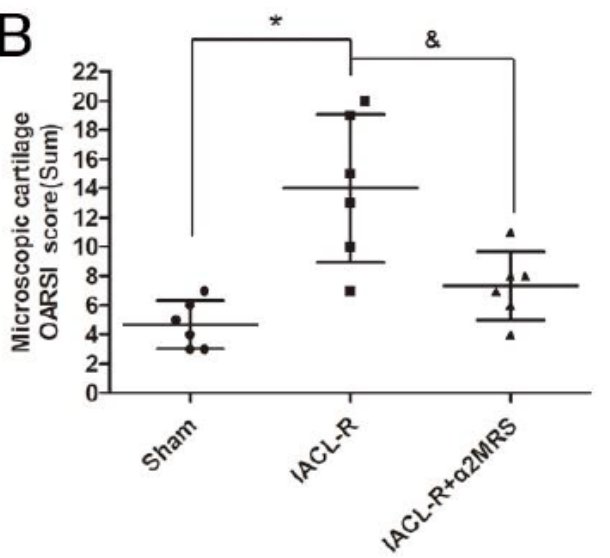

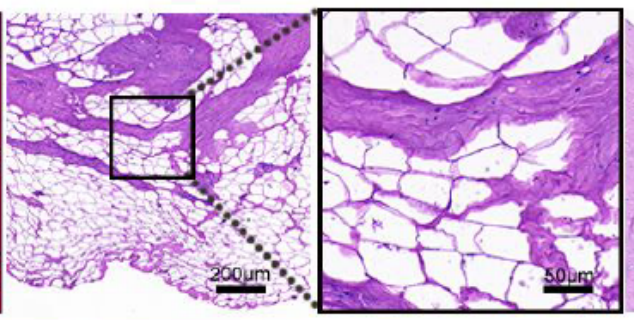
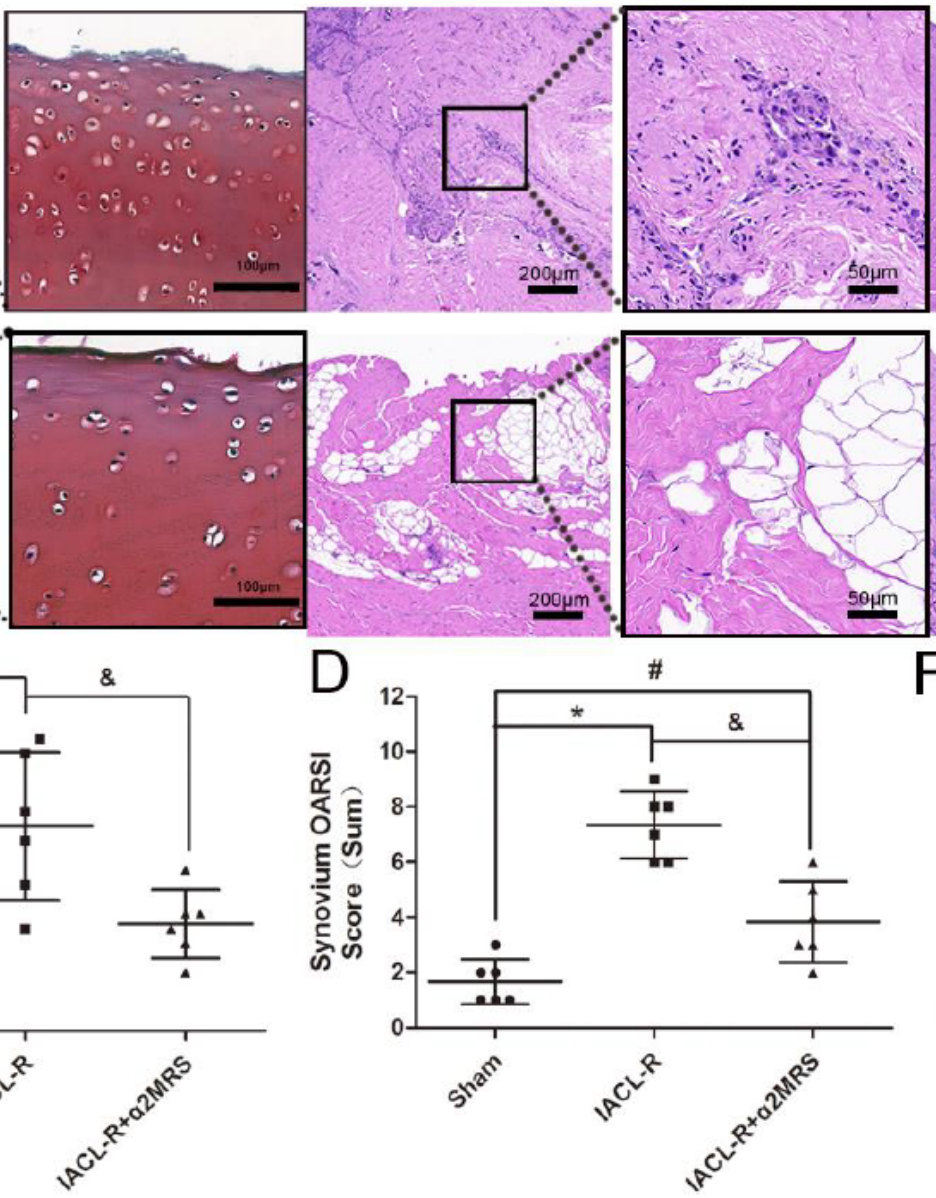

$E$

Meniscus (H\&E)
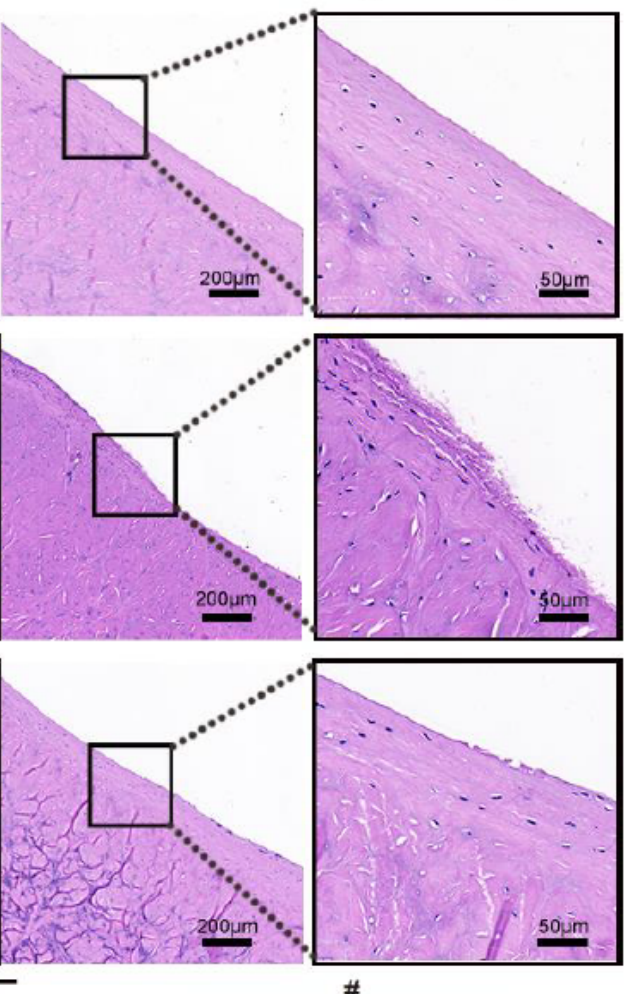

$\mathrm{F}$

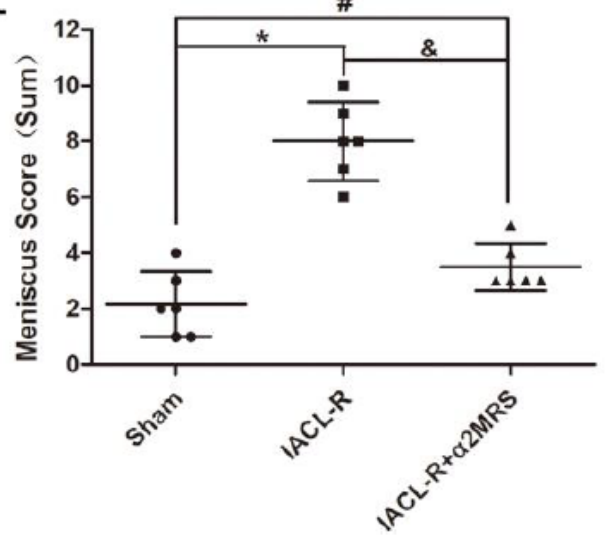

Figure 8

A: Compared with the IACL-R group, less decrease in safranin $O$ staining and surface fibrillation were observed in the IACL-R+a2MRS group. B: Microscopic cartilage score were lower in the IACL-R+a2MRS group than in the IACL-R group $P=0.015)$. C: On H\&E staining of the synovium, we found less degeneration in the IACL-R+a2MRS group than in the IACL-R group. D: Microscopic synovium score were lower in the IACL-R+a2MRS group than in the IACL-R group $(P=0.002)$. E: On H\&E staining of the meniscus, we found less degeneration in the IACL-R+a2MRS group than in the IACL-R group F: Microscopic meniscus score were lower in the IACL-R+a2MRS group than in the IACL-R group $(P<0.001)$. $*=P<0.05$, sham group versus IACL- $R$ group; \#=P<0.05, sham group versus IACL-R+a2MRS group; $\&=P<$ 0.05 , IACL-R group versus IACL-R+a2MRS group. The bars show the mean $\pm S D(n=6)$. 
$\begin{array}{llllll}\text { A Col-2 } & \text { MMP-3 } & \text { MMP-9 } & \text { MMP-13 } & \text { Col-10 } & \text { Runx-2 }\end{array}$
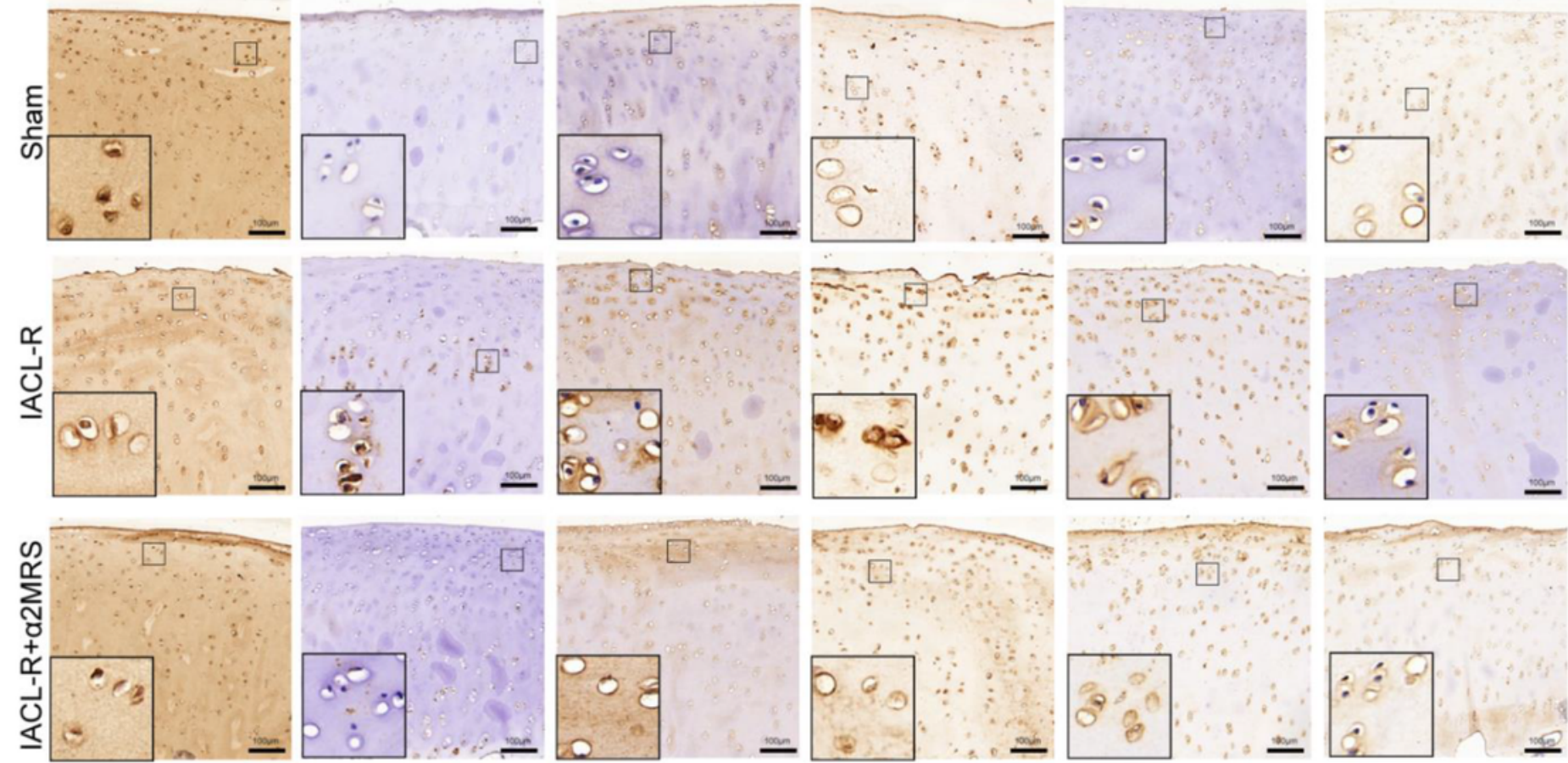

C MMP-3(Synovium)

MMP-9(Synovium) MMP-13(Synovium) B
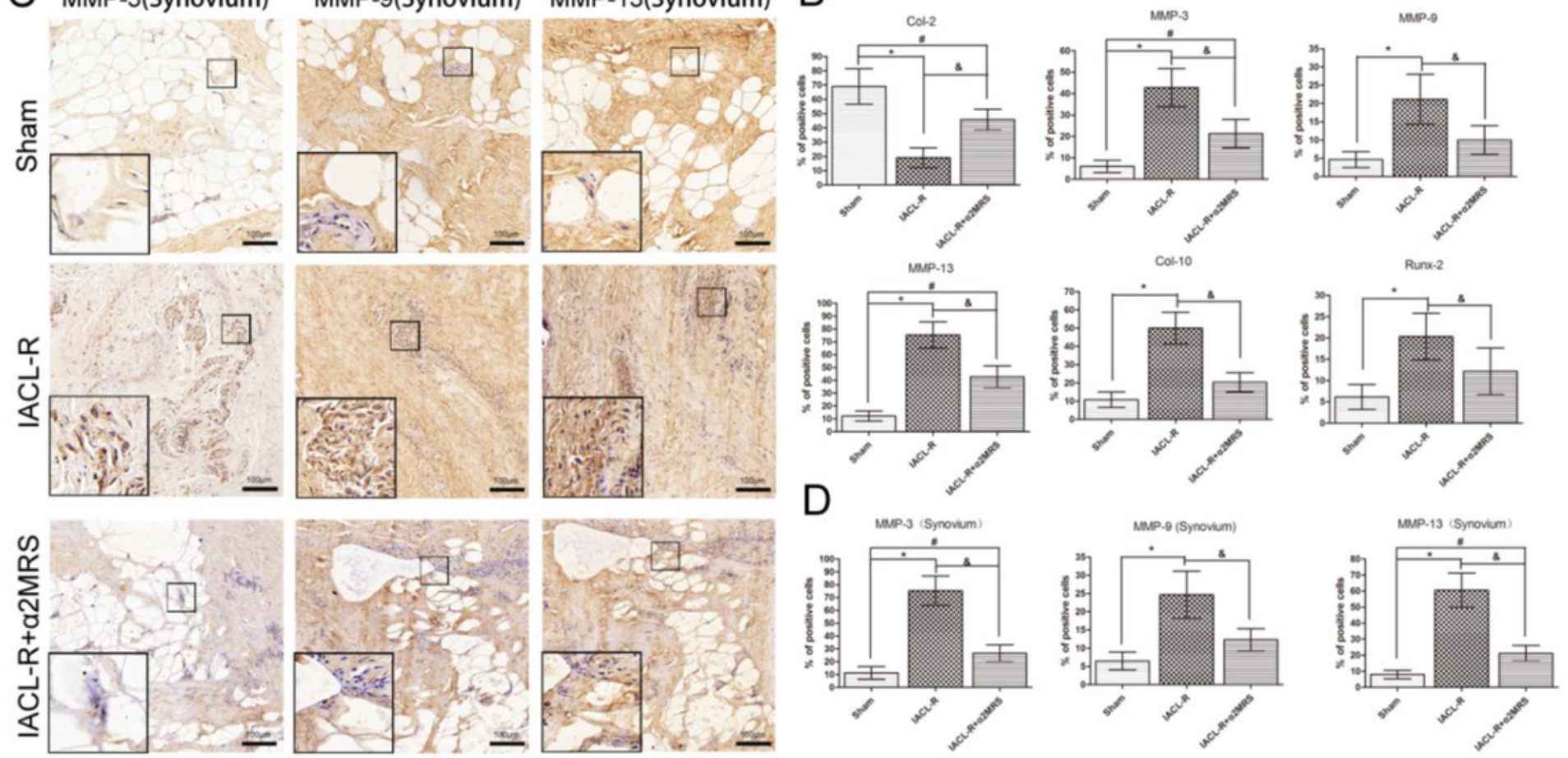

\section{Figure 9}

A: Articular cartilage immunostaining showed that MMP-3, MMP-9, MMP-13, Runx-2, and Col -10 staining significantly increased in the IACL-R mini pig group but were lower in the IACL-R+a2MRS group. In contrast, Col-2 expression in articular cartilage was higher in the IACL-R+a2MRS group than in the IACL-R group. B: Quantitative immunohistochemical analysis of articular cartilage. C: synovium immunostaining showed that MMP-3, MMP-9 and MMP-13 staining significantly increased in the IACL-R mini pig group 
but were lower in the IACL-R+a2MRS group. D: Quantitative immunohistochemical analysis of synovium. $*=P<0.05$, sham group versus IACL-R group; \#=P<0.05, sham group versus IACL-R+a2MRS group; $\&=P<$ 0.05 , IACL-R group versus IACL-R+a2MRS group. The bars show the mean $\pm S D(n=6)$

\section{Supplementary Files}

This is a list of supplementary files associated with this preprint. Click to download.

- Supplementaryfigure.docx

- Supplementarytable.docx

- Supplementarytext.docx 